\title{
Dynamic stem cell states: naïve to primed pluripotency in rodents and humans
}

\section{Leehee Weinberger, Muneef Ayyash, Noa Novershtern \& Jacob H. Hanna}

The Department of Molecular Genetics, Weizmann Institute of Science, Rehovot 7610001, Israel.

Correspondence to: J.H.H (Jacob.hanna@weizmann.ac.il) and N.N

(noa.novershtern@weizman.ac.il).

\section{Preface}

The molecular mechanisms and signalling pathways that regulate the in vitro preservation of distinct pluripotent stem cell configurations, and their induction in somatic cells via direct reprogramming approaches, continue to constitute a highly exciting area of research. In this review, we provide an integrative synthesis on recent discoveries related to isolating unique naïve and primed pluripotent stem cell states with altered functional and molecular characteristics, and from different species. We overview pathways underlying pluripotent state transitions and interconversion in vitro and in vivo. We conclude by highlighting unresolved key questions, future directions and potential novel applications of such dynamic pluripotent cell states.

\section{Introduction}

Pluripotency describes cells that have the potential to give rise to cells from all three embryonic germ-layers and possibly to primordial germ cells (PGCs), but not extra-embryonic tissues ${ }^{1}$. While pluripotency is a transient state in vivo, pluripotent cells can be derived from different stages of early embryonic development and indefinitely maintained in an artificially induced self-renewal state in vitro, by supplementing exogenous cues ${ }^{2}$. Thus, it is important to stress that self-renewal is not a defining feature of pluripotency and is only transiently assembled during early development. Pluripotency is highly dynamic and evolves at different stages of pre- and post-implantation stages ${ }^{3}$. However, the self-renewal aspect is a highly useful in vitro artificial "engineering trick" that has brought pluripotent cells to the front stage as a tool for tissue replacement, disease modelling and animal engineering technologies 5 .

There are multiple pluripotent stem cell types that can be isolated from vertebrates, including rodents and human, typically annotated based on their donor cell-of-origin (Fig. 1). Embryonic stem cells (ESCs) are isolated from the inner cell mass (ICM) of developing pre-implantation mouse or human blastocysts ${ }^{6-8}$. Epiblast stem cells (EpiSCs) are isolated from mouse postimplantation epiblasts 9,10 , however equivalent derivations haven't been attempted with human embryos due to justified ethical complexities. Early rodent migrating PGCs can be converted in vitro into pluripotent ESC-like cells termed embryonic germ cells ${ }^{11,12}$. Mouse neonatal and adult spermatogonial stem cells can be reverted toward pluripotency and generate male germ stem cells (GSCs) ${ }^{13-15}$. The latter have the disadvantage of retaining only male imprint signature, which can increase tumorigenic potential ${ }^{15}$. Intriguingly, stable and validated EGs and GSCs have not been isolated from primates thus $\operatorname{far}^{16,17}$ (Fig. 1).

Somatic cell reprogramming provides alternative routes for isolating pluripotent cell types. Human and rodent somatic cells can be artificially reprogrammed into ESC-like cells following reprogramming via nuclear transfer, termed NT-ESCs ${ }^{18-20}$. Ten years ago, Yamanaka established direct in vitro reprogramming of somatic cells to pluripotency via ectopic expression of defined 
factors $^{21}$, that yield induced pluripotent stem cells (iPSCs) without the need for oocytes or embryos $^{22-25}$ (Fig. 1). NT-ESCs and iPSCs offer the advantage of being able to generate patient specific pluripotent cells with nuclear DNA identical to the donor somatic cell, however mitochondrial DNA in NT-ESCs is non-isogenic and provided by the a-nucleated donor oocytes $^{26}$. The latter can be an advantageous in settings aiming at correcting maternally inherited mitochondrial diseases ${ }^{27-29}$.

While the above overview pertains to classify different pluripotent cell types based on their tissue derivation source, the growth conditions used to expand such cells dictate the pluripotent state they attain (ICM-like, ESC-like, EpiSC-like state etc.) ${ }^{30,31}$. iPSCs generated in classical mouse ESC growth conditions yield ESC-like iPSCs; while those reprogrammed in EpiSC growth conditions yield EpiSC-like iPSCs ${ }^{30,32}$. The same analogy applies to explanting rodent ICM cells in ESC or EpiSC growth conditions ${ }^{30,33}$. In comparison to developmentally restricted mouse EpiSCs, ESCs are highly competent in generating high-contribution chimeric mice after microinjection into host-blastocysts, retain a pre x-inactivation state in female cell lines and reduced expression of lineage commitment factors ${ }^{30,31}$. Such attributes influence the utility of pluripotent cells in cell differentiation and animal transgenics. Thus it is of importance to understand and define different pluripotent states and configurations across different species ${ }^{4}$.

In this review we provide an integrative perspective on recent breakthroughs in understanding the diversity and complexity of pluripotent state regulation in vitro. This includes advances on preserving naïve pluripotency from non-rodent species and alternative pluripotent states. We highlight unresolved issues, key questions and future directions in this exciting front of stem cell research.

\section{Murine pluripotent states}

Mouse ESCs were shown to reside in an ICM-like state ${ }^{30}$, referred to as the naïve state of pluripotency $^{31}$, since they retain several molecular characteristics of ICM. A novel type of pluripotent cells, termed EpiSCs, was later derived from post-implantation rodent epiblasts ${ }^{9,10}$. In comparison to naïve ESCs, EpiSCs retain an alternative pluripotency configuration, referred to a primed pluripotency ${ }^{31}$. Profound molecular and functional differences exist between different pluripotent cells, which subsequently influence their characteristics, function and safety.

\section{Growth conditions for naïve pluripotency}

To grasp the biology of mouse naïve ESCs and their developmental context, it is of relevance to review the evolution of growth conditions devised to isolate such cells over the course of the last thirty years. Martin and Evans derived ESCs from 129 -mouse strain ${ }^{7,8}$, by utilizing mitotically inactive embryonic fibroblast cells (MEFs) as feeder cells and foetal bovine serum (FBS) ${ }^{34}$. Leukaemia Inhibitory Factor (LIF) that activates the STAT3/JAK pathway was later identified as a key ingredient that allowed expansion of mouse ESCs in FBS/LIF conditions without $\mathrm{MEFs}^{35,36}$ (Fig. 2). Such naïve mouse ESCs express hallmark pluripotency factors (e.g. Oct4, Nanog, Esrrb) and retain a pre-x inactivation state in female cell lines ${ }^{37}$ (Fig. 3). Functionally, ESCs can populate the host pre-implantation mouse ICM upon microinjection into blastocysts, and generate high-contribution chimeras with colonization of the germ-line ${ }^{37}$.

Smith group described the first serum- and feeder-free defined conditions for expanding mouse ESCs by combining low-dose BMP4 with LIF $^{38}$. Addition of small molecule inhibitors for MEK signaling increased ESC derivation efficiency and stability ${ }^{39}$. Developing different defined conditions, all involving MEK inhibitors, which can be used to isolate murine ESCs, extended the latter defined growth conditions. A cocktail combing 3 inhibitors termed ' $3 i$ conditions', was shown to stabilize pluripotent cells without LIF, indicating existence of redundant pathways to 
isolate ESCs in vitro that can compensate for lack of LIF/Stat3 signalling ${ }^{40}$. Notably, such cell configuration was labelled as "ground state pluripotency" since the cells in $3 \mathrm{i}$ were reported to grow independent of any exogenous signalling stimuli. However, this term is challenged by the fact that growth in these conditions heavily relies on GSK3 inhibition that mimics WNT stimulation; and on exogenous insulin that activates PI3K/AKT signalling ${ }^{40}$. Further, 2i/LIF conditions were adopted as an enhanced mean to expand murine ESCs, and the reduced proliferation in $3 \mathrm{i}$ conditions indicates a role for autocrine secreted FGF4 in promoting naïve ESC growth independent of $\mathrm{Mek} / \mathrm{Erk}^{30,41,42}$ (Fig. 2). Notably, complete genetic ablation of Erk1/2 is indispensable for maintaining rodent naïve ESC survival and does not yield an identical phenotype to Mek inhibition, suggesting additional roles for Mek inhibition in maintaining ESCs stability that are Erk independent ${ }^{43}$.

"Alternative 2i" conditions, involving small molecule inhibitors for Gsk3 and Src pathways, yield germ-line competent $\operatorname{ESCs}^{44}$ (Fig. 2). Atypical PKC small molecule inhibitor Go6983 (aPKCi) was identified as another stimulator for isolating murine ESCs together with LIF and/or MEK inhibitors ${ }^{45}$. Single cell RNA-seq analysis has proven equivalent global heterogeneity between different naïve conditions, however the difference exists in the identity of genes that underlie heterogeneity in each condition ${ }^{46}$.

Enriched conditions were important for deriving ESCs from other mouse strains that have until recently been considered "non-permissive" for deriving naïve ESCs. While 129-mouse strain derived ESCs could be expanded in FBS/LIF conditions, for other mouse strains like non-obesediabetic mice (NOD), supplementation of $2 \mathrm{i}$ or GSK3 inhibitors is essential for both derivation and maintenance ${ }^{30,42} .3 \mathrm{i}$ and $2 \mathrm{i} / \mathrm{LIF}$ conditions yielded rat ESCs, however these conditions are suboptimal $^{47,48}$. LIF/MEKi/aPKCi constitute a more robust method to support rat ESCs ${ }^{49}$ (Fig. 2,3).

The above findings underscore the relevance of analysing rodent ESCs expanded in different naïve conditions and from different genetic backgrounds. Further they emphasize the importance of other signalling pathways that remain to be deeply characterized in the context of pluripotency (Fig. 2). SRC functions as a downstream target of MEK/ERK and Calcineurin-NFAT signalling to promote ESC differentiation ${ }^{50}$, and its inhibition promotes naïve pluripotency ${ }^{44,50}$. NF-kB inhibition has been implicated as a downstream effector underlying aPKC inhibition mediated support of naïve pluripotency ${ }^{45}$, however other pathways, like Mbd3/NuRD repressor complex, can be neutralized by aPKCi (unpublished observations, J.H.H).

Hippo signalling pathway regulates epiblast vs. trophoblast segregation in late mouse morulas, and is highly active in pluripotent epiblast cells, leading to exclusion of Yap/Taz effectors from the nucleus ${ }^{51,52}$. Piccolo and colleagues indicated that Yap/Taz depletion in mouse naïve ESCs expanded in 2i/LIF further enhances their resistance to differentiation ${ }^{52}$, while Lian et al. have indicated Yap/Taz as essential regulators for stability of naïve ESCs expanded in FBS/LIF ${ }^{53}$. Reanalysis of these findings in different conditions might resolve these seemingly opposing results (Fig. 3).

It should be noted that signalling pathways are often pleiotropic and may simultaneously have positive and negative effects on naïve pluripotency. For instance, nuclear $\beta$-catenin stabilization following Gsk3 inhibition, promotes naïve pluripotency via neutralizing the repressive activity of Tcf3 on its bound target genes in the nucleus ${ }^{54}$. Cytoplasmic $\beta$-catenin promotes naïve pluripotency via increasing E-cadherin membrane stability ${ }^{55}$ (Fig. 2). However, nuclear $\beta$ catenin can induce mesodermal gene expression through its Lef co-effectors ${ }^{47}$. Yet, such differentiation priming effects are outweighed by naïve pluripotency promoting functions of $\beta$ - 
catenin under optimized conditions. LIF has also been shown to promote primitive endoderm specification in naïve pluripotency growth conditions ${ }^{56}$. Such "non-purist" effects should be kept in mind when dissecting the role of signalling pathways on pluripotency.

The ample conditions to grow naïve murine ESCs have been important for better understanding and revisiting the roles of several classical pluripotency regulators. While Nanog was first purported to be absolutely essential and irreplaceable for establishing naive pluripotency through iPSC reprogramming, cell fusion or EpiSC reversion ${ }^{57}$, multiple conditions enable reprogramming of Nanog null donor cells in vitro ${ }^{58-60}$. Still however, Nanog null ESCs cannot be derived from mouse ICMs, indicating that while Nanog is indispensible for establishing pluripotency in vivo, it is dispensable during in vitro induction ${ }^{61}$. The latter example highlights that in vitro pluripotency maintenance cannot be considered "authentic", as some in vitro conditions can potentiate the robustness of the naïve pluripotency program and compensate for deficiencies that are not sustainable in vivo. Similarly, Klf2 knockout embryos do not present lethality at the pre-implantation stage, and naïve ESC in 2i/LIF or FBS/LIF conditions can tolerate Klf2 ablation ${ }^{62}$. However ESCs in $2 \mathrm{i}$ only conditions, cannot sustain loss of Klf $2^{62}$, as LIF can compensate for the lack of Klf2.

Another emerging regulatory principle is that not all factors expressed in the ICM or ESCs necessarily promote naïve pluripotency, and some of them in fact are promoting its dissolution. However, they are tolerated by ESCs in vitro due to the optimized and enriched in vitro growth conditions used. For instance, Tcf3 binding represses the expression of its naïve pluripotency promoting target genes, leading to their partial repression but is tolerated in FBS/LIF naïve conditions. However, Tcf3 neutralization by adding GSK3i boosts naïve pluripotency ${ }^{54}$. In a similar manner, mouse ESCs tolerate Mbd3/NuRD complex expression although it partially represses naïve pluripotency targets ${ }^{63}$. However, genetic ablation of Mbd3 leads to upregulation of master regulators of naive pluripotency and allows LIF independent growth ${ }^{63}$. Consistently, derivation of Mbd3 KO ESCs from ICMs is uncompromised in 2i/LIF conditions ${ }^{64}$. In summary, both Tcf 3 and Mbd3 are expressed in the ICM and ESCs, likely to set the stage for terminating naïve pluripotency. Thus, the molecular characteristics of a pluripotent state fixated in a certain condition represent the net outcome of conflicting stabilizing and destabilizing factors simultaneously residing and conflicting in that state ${ }^{65}$.

Collectively, it is important when analysing function of pluripotency regulators to systematically compare different naïve growth conditions, genetic backgrounds and in vivo context ${ }^{66}$. Such integrative analysis will likely unravel additional layers of underappreciated complexity and may resolve some conflicting results ${ }^{52,53}$.

\section{Growth conditions for primed pluripotency}

Primed EpiSCs were derived from post-implantation epiblasts of rodents in Fgf2/Activin A conditions $^{9,10}$ (Fig. 1). EpiSCs are capable of differentiating into cells of all three germ layers in vitro or in teratoma assay, and thus are pluripotent. However, they are inefficient in yielding chimeric animals once injected in pre-implantation epiblasts (Fig. 3), likely because they have altered molecular characteristics and correspond to a more advanced developmental stage in comparison to the host pre-implantation environment ${ }^{9,10}$. They can however form low contribution chimeric embryos, when injected into host-post implantation embryos ex-vivo ${ }^{67}$.

While EpiSCs maintain Oct4 and Sox2 expression, they down-regulate most of the other pluripotency factors including Nanog, Esrrb, Klf2 and $\mathrm{Klf}^{3}$. EpiSCs have not undergone differentiation, but they upregulate lineage commitment factors like Otx2, Brachyury and Zic2 ${ }^{68}$. Epigenetically, EpiSCs retain distinct characteristics from naïve ESCs: they inactivate $\mathrm{X}$ 
chromosome in females, upregulate global DNA methylation levels and acquire H3K27me3 at developmental regulators ${ }^{69,70}$. Enhancer landscape is rewired between naive and primed states ${ }^{68}$, and developmental regulator gene associated "seed enhancers" convert from a dormant to an active state in EpiSCs, thus pre-marking lineage differentiation bias of primed PSCs ${ }^{71}$. Summary of divergent signalling and molecular characteristics between murine primed and naïve cells are highlighted in Fig. 2-3.

At the regulatory level, naïve and primed pluripotent cells have been shown to retain opposing dependence on epigenetic repressors ${ }^{66}$ (Fig. 4). Naïve ESCs expanded in FBS/LIF or 2i/LIF tolerate loss of epigenetic repressors like Dnmt1, Dicer, Eed, Mbd3 and Mettl3 ${ }^{66}$, and renders these cells "hyper-naïve" and resistant to differentiation ${ }^{66,72}$ (Fig. 4). On the contrary, murine primed pluripotency maintenance and viability depends on these regulators, and their ablation destabilizes the murine primed pluripotent state ${ }^{66}$ (Fig. 4). Defining how the depletion of each of these repressors precisely destabilizes the primed configuration is of future interest.

Alternative growth conditions to expand murine EpiSCs have begun to emerge. Ying and colleagues showed that simultaneous use of a GSK3 inhibitor (that induces $\beta$-catenin stabilization), together with a Tankyrase small molecule inhibitor IWR1 (that up regulates Axin $1 / 2$ levels thus leading to retention of $\beta$-catenin in the cytoplasm) maintain novel primed EpiSCs without exogenous Fgf2/Activin A supplementation ${ }^{73}$ (Fig. 3). Removal of IWR1 leads to increased nuclear $\beta$-catenin shuttling and EpiSC differentiation ${ }^{73}$. The mechanisms by which cytoplasmic $\beta$-catenin prevents EpiSC differentiation remains to be uncovered ${ }^{73}$. It is tempting to speculate whether the recently described ability of cytoplasmic APC/Axin/ $\beta$-catenin destruction complex to act as a sequestration "sink" for Yap/Taz and prevent their nuclear shuttling ${ }^{52}$, is involved in the ability of GSK3i/IWR1 conditions to maintain EpiSCs. Notably, the latter alternative EpiSC state is different from EpiSCs expanded in classical Fgf2/Activin A conditions, and retains higher expression of naïve markers ${ }^{73}$, and are thus relatively "less primed" (Fig. 3).

Recent studies indicate that different primed conditions can endow EpiSCs with region specific characteristics of the post-implantation epiblasts. EpiSCs expanded in Fgf2/Activin A correspond transcriptionally and functionally to anterior late-gastrula primitive streak cells ${ }^{74}$. Alternative FGF2/IWR1 conditions generate murine EpiSCs corresponding rather to posteriorproximal epiblasts ${ }^{75}$. Further, even in classical Fgf2/Activin A conditions distinct subpopulation of EpiSCs can co-exist, each representing different stages of post-implantation embryonic development ${ }^{76}$.

Finally, the time length at which pluripotent cells are maintained under primed conditions greatly influences their characteristics and functionality ${ }^{77}$. Counter intuitively; while murine PGCs are specified from the post-implantation epiblast in vivo, EpiSCs maintained in vitro for more than 7 days in FGF2/Activin A, lose competence to generate PGCs in response to Bmp4 ${ }^{77}$. Starting with naïve cells and inducing brief priming for 2-4 days, yields distinct primed cells highly competent for generating PGCLCs, termed EpiLCs ${ }^{77}$. The latter are transcriptionally more similar to in vivo post-implantation epiblast than EpiSCs ${ }^{77}$. Thus, the above paradigm indicates another aspect of artificial features that can be acquired by pluripotent cells once expanded indefinitely in vitro, in contrast to them in vivo "counterparts" that transiently exist during development.

Studies involving clonal lines and single cell analysis will provide deeper understanding of features of region specific EpiSCs and shortly after in vitro induction from a naïve state in different priming conditions ${ }^{74}$. This may help understand how lineage priming is established at 
the single cell level during these key early developmental transitions ${ }^{74,75}$ and might be relevant for optimizing other differentiation protocols and predicting PSC behaviour.

\section{Interconversion between naïve and primed states}

As somatic cells can be reprogrammed into a naïve ESC-like state via combined overexpression of pluripotency factors together with LIF, primed EpiSCs can also be reverted to naive iPSCs. Overexpression of Klf4 or Myc in EpiSCs, under LIF containing conditions, generates naïve $\mathrm{ESCs}^{30,78}$. FBS/LIF signalling alone can be sufficient to induce such conversion from permissive mouse genetic backgrounds (i.e. 129 strains) ${ }^{79}$, but not from "non-permissive" ones like NOD, where supplementation of small molecules like $2 \mathrm{i}$ is necessary ${ }^{30}$. Other factors like Nanog, Prdm14 and Esrrb have been shown to synergistically induce and boost the efficiency of this process $^{80,81}$. Explanting post-implantation E5.5-E7.5 epiblasts in naïve conditions also reverts them into naïve PSCs ${ }^{30,79}$. The opposite conversion is attainable from in vitro and in vivo isolated naïve cells, as expanding murine naïve PSCs or ICMs in primed conditions leads them to gradually adapt an EpiSC state ${ }^{30,33,78}$.

Studies focusing on in vitro molecular changes accompanying naïve to primed pluripotency conversion have unravelled key events in understanding mechanisms of reprogramming ${ }^{69}$. Naïve ESCs expanded in 2i/LIF retain global hypo-methylated levels in both promoters and gene bodies, highly similar to those measured in $\mathrm{ICMs}^{82,83}$. When transferred into LIF/FBS naïve conditions, this is accompanied by an increase in global DNA methylation levels, however promoter and enhancer regulatory regions remain protected from invasion by DNA methylation $^{84}$. Only after transfer into primed Fgf2/Activin A EpiSC inducing conditions, DNA methylation accumulates over enhancer and promoter regulatory elements ${ }^{84}$.

Transitioning naïve FBS/LIF PSCs into 2i/LIF conditions leads initially to changes in Oct4, Sox 2 and Nanog pluripotency factor occupancy ${ }^{85}$. Changes in H3K27me3 deposition and enhancer landscape follows later, likely in response to the rewiring in transcription factor binding ${ }^{85}$. Downregulation in DNA methylation follows next, which has mainly been attributed to downregulation in de novo DNA methyltransferase enzymes ${ }^{82}$. It should be noted however, that ablation of Dnmt3a/b in ESCs in FBS/LIF condition does not lead to such rapid loss of DNA methylation $^{86}$, and other yet to be identified events might be involved in this rapid $2 \mathrm{i}$ induced epigenetic response. MEK/ERK inhibition influences polycomb interactions and leads to decreased occupancy by PRC2 and decreased phosphorylation on the CTD of PolII on lineage commitment genes ${ }^{87}$, leading to loss of H3K27me3 and increased PolII pausing at bivalent developmental regulatory loci ${ }^{69}$. Analysing other defined naïve pluripotency growth conditions ( $2 \mathrm{i} / \mathrm{LIF} / \mathrm{PKCi}$, alternative $2 \mathrm{i}$ etc.) and in other rodents, will be important for discerning redundancies and specificities of different singling pathways and how they cross-talk with chromatin organization.

\section{Human conventional pluripotent cells}

Thomson group first isolated human ESCs from bastocysts ${ }^{6}$. Surprisingly, they were drastically different from murine ESCs in their characteristics and tissue culture requirements. FGF2 and TGF $\beta 1 /$ ACTIVIN, but not LIF, signalling are at the core signalling modules maintaining such conventional human ESCs derived from the ICM, or iPSCs obtained via direct in vitro reprogramming ${ }^{88}$.

\section{A primed pluripotent state}

Differences between conventional human and mouse ESCs had been initially attributed only to unknown species genetic differences, since human ESCs were also derived from the ICM and not from post-implantation stages. However studies on different mouse strain derived stem cells 
have discerned a scenario where ICM cells can adapt in vitro into a primed state if naïve conditions that match the requirements of the particular genetic background of donor embryos used, are not devised ${ }^{30}$. Specifically, NOD mice are relatively "less-permissive" than 129 mice to yield naïve ESCs/iPSCs, as LIF alone is not sufficient to maintain NOD naïve pluripotency and $2 \mathrm{i} / \mathrm{LIF}$ are permanently required to stabilize and maintain this state in vitro in NOD PSCs ${ }^{30}$. Further, ICMs from both 129 and NOD strain expanded in primed conditions yield EpiSCs that are indistinguishable from EpiSCs derived from E6.5 embryos $^{30}$ or in vitro from already established $\mathrm{ESCs}^{30}$.

The relevance of the latter in vitro priming scenario to dictating conventional human ESC identity is supported by the fact that human conventional ESCs/iPSCs retain a great milieu of primed pluripotency features. This includes low expression of naïve pluripotency markers (e.g. TFCP2L1, DPPA3), deposition of H3K27me3 over developmental genes, lack of exclusive nuclear localization of TFE3, loss of pluripotency upon inhibition of MEK/ERK pathway, lack of global hypomethylation as seen in ICM cells, lack of a pre x-inactivation state in most conventional female PSCs lines ${ }^{70,89,90}$. Further, human primed ESCs do not tolerate complete loss of DNMT1 ${ }^{86}$, similarly to what has been shown for mouse EpiSCs ${ }^{66}$ (Fig. 4). Complete human KO ESCs have not been obtained thus far for DICER, MBD3 or METTL3 ${ }^{64,66}$ (Fig. 3).

\section{Less primed than murine EpiSCs}

In spite of the above, it is of importance to realize that human conventional/primed ESCs are not identical to murine EpiSCs, and can be considered relatively "less primed". For instance, human ESCs do not upregulate FGF5 and N-CADHERIN as seen in murine EpiSCs, and express highlevels of E-CADHERIN as detected in mouse naïve ESCs ${ }^{70}$. Human ESCs express high levels of some naïve markers like NANOG, PRDM14 and REX1 that are residually expressed in murine EpiSCs ${ }^{91}$. Moreover, human primed ESCs are functionally dependent on NANOG and PRDM14 and their ablation induces differentiation ${ }^{91}$. DNA methylation distribution in human ESCs shows they rather correspond to murine naïve ESCs expanded in FBS/LIF conditions, rather than mouse FGF2/Activin A expanded mouse EpiSCs, as their promoters are protected from invasion by repressive DNA methylation ${ }^{84,92}$. Further, while murine EpiSCs demonstrate exclusive TFE3 cytoplasmic localization and naïve 2i/LIF ESCs show exclusive nuclear TFE3 localization ${ }^{93}$, human primed ESCs show an intermediate configuration where TFE3 is present in both the cytoplasm and the nucleus ${ }^{70}$.

\section{Human naive pluripotent cells}

The metastability of naïve and primed pluripotent state depending on the growth conditions applied $^{30}$, and the stringency in requirement for exogenous naïve pluripotency promoting factors to isolated naïve PSCs from previously "non-permissive" rodent strains ${ }^{30,31}$, have underscored a scenario of whether unique and more stringent conditions can be applied to isolated previously unidentified alternative naïve-like pluripotent states in humans.

\section{Transgene-dependent generation}

2i/LIF conditions are not sufficient to maintain naïve human ESCs or iPSCs ${ }^{94}$. However, additional transgene expression can induce an artificial transgene dependent state that may be of considerable interest. Continued exogenous OCT4/KLF4 or KLF2/KLF4 transgene expression can maintain human ESCs/iPSCs in a unique pluripotent state in $2 \mathrm{i} / \mathrm{LIF}$ conditions ${ }^{94}$. Recently, these observations were extended by optimizing over-expression of KLF2/NANOG transgenes, allowing expansion of human naïve iPSCs in $2 \mathrm{i} / \mathrm{LIF}^{95}$. Smith and colleagues overexpressed KLF2 and NANOG transgenes in primed ESCs and expanded them in $2 \mathrm{i} / \mathrm{LIF} / \mathrm{aPKCi}$ conditions 96. These cells exhibited extensive DNA hypomethylation, and strong upregulation of naïve markers like TFCP2L1, KLF2, and KLF4. However, as KLF2 is not expressed in the human 
$\mathrm{ICM}^{97}$, and as $2 \mathrm{i} / \mathrm{LIF} / \mathrm{aPKCi}$ are insufficient to convert primed ESCs without exogenous transgene induction $^{96}$, and as transgene-free cells remain to be validated in $2 \mathrm{i} / \mathrm{LIF} / \mathrm{aPKCi}$ conditions, it is unclear whether this state is indefinitely stable without retaining possibly leaky transgenes or MEFs. Further, independent examination of DNA methylation landscape in these cells indicates aberrant global loss of imprinting, excessive hypomethylation of endogenous retroviral genes ${ }^{89,98}$. Finally, while $2 \mathrm{i} / \mathrm{LIF} / \mathrm{aPKCi}$ conditions do not contain exogenous FGF or TGF $\beta 1 /$ Activin A cytokines, applying only short-term inhibition of FGFR/TGFR signalling is not sufficient evidence to validate FGF/TGF/Activin A signalling independence ${ }^{96}$ (Fig. 2). Indeed, unlike in mice, human pluripotent ICM collapses upon treating blastocysts with small molecule inhibitors for TGF/ACTIVIN signalling ${ }^{97}$.

While the field has shifted to study transgene independent conditions as detailed below, it should be noted that such transgene dependent states might be important, since it may be possible that robust naïve pluripotency currently obtained in mouse ESCs is a rodent specific phenomenon. Capturing human naive PSCs identical to those obtained from mice might still involve genetic modifications. Nevertheless, the latter studies provide evidence for the possibility to generate alternative pluripotent states in humans and other species ${ }^{30,94}$.

\section{Transgene independent generation}

Our team was the first to describe naïve conditions, designated as NHSM (Naïve Human Stem cell Medium), which entail complete ablation of MEK/ERK signalling and are compatible with indefinitely expanding genetically unmodified human PSCs both in MEF- containing and -free conditions $^{70}$. These naïve MEK independent pluripotent cell lines could be derived from human pre-implantation embryos, through de novo iPSC generation, or from previously established primed ESCs/iPSCs ${ }^{70}$. NHSM conditions contain $2 \mathrm{i} / \mathrm{LIF}$ together with P38i, JNKi, aPKCi, ROCKi, low doses of FGF2 and TGF $\beta 1$ (or Activin A), and render human PSCs more similar, but not identical, to murine naïve $\operatorname{PSCs}^{70}$ (Fig. 2,3). In fact, these cells have features of so called naïve $2 \mathrm{i} / \mathrm{LIF}$ "ground state" pluripotency, which are not found even in naïve mouse ESCs expanded in FBS/LIF. This includes exclusive nuclear localization of TFE3, and cleansing of H3K27me3 over developmental genes ${ }^{69,70,93}$. Transcriptionally, these cells down regulated expression of lineage commitment markers like OTX2, ZIC2 and CD24 and moderately upregulated pluripotency genes (more prominently on MEFs and when aPKCi is used) ${ }^{70,99}$. Enhancer rewiring has been attained in these human naive PSCs as seen with mouse cells ${ }^{71}$. The cells exhibited downregulation in DNMT3B ${ }^{100}$ and a mild global decrease in global DNA methylation levels, while maintaining imprinting integrity and chromosomal stability ${ }^{70}$. While conducting chimeric analysis with human PSCs and using human embryos as hosts is ethically and legally forbidden, these human naïve PSCs cells showed better integration into blastocysts upon microinjection into host mouse morulas and were able to contribute at low-grade levels in mouse embryos up to E10.5-E17.5 ${ }^{70}$ (Fig. 3).

Important publications describing alternative conditions that yield human MEK independent naïve pluripotent cells emerged after, each producing cells with different enhanced molecular properties (Fig. 3). A combination of 2i/LIF, ROCKi, BMPRi, high doses of FGF2 and TGFB1 were able to maintain human PSCs only in the presence of MEFs ${ }^{101}$. These cells demonstrated transcriptional upregulation of pluripotency markers like STELLA and KLF5. Jaenisch team described conditions ${ }^{95}$ that adopted most of components found in $\mathrm{NHSM}^{70}$ (i.e. 2i/LIF, ROCKi, Activin A (instead of TGF $\beta 1$ ) - with or without FGF2 and JNKi) and supplemented inhibitors for BRAF and SRC pathways (conditions termed 5i/LA-MEF with optional inclusion of JNKi or FGF2). In comparison to previous studies, cells in 5i/LA-MEF conditions demonstrated a more impressive upregulation of naïve pluripotency markers. However, the cells did not down regulate DNMT3B, maintained an inactive X chromosome state in female cell lines, and demonstrated an 
unusual pre-ICM signature ${ }^{95}$. Intriguingly, the process of converting primed cells back to naïve state in 5i/LA-MEF conditions is inefficient, taking two weeks to isolate initial clones that retain a slow growth rate ${ }^{95}$. Further, these conditions exclusively yield chromosomally abnormal cell lines ${ }^{95}$. Thus, it remains to be determined whether such chromosomal abnormalities are in fact inherent to $5 \mathrm{i} / \mathrm{LA}-\mathrm{MEF}$ cells and dictate the properties described for this state ${ }^{95}$, and are being selected for during this inefficient conversion process. Finally, DNA methylation profiling and whether these cells maintain epigenetic imprinting integrity is another important aspect that remains to be evaluated.

It is clear from the above summaries that none of the many already published conditions generate human naïve PSCs that are identical to mouse ESCs or human $\mathrm{ICM}^{97,102,103}$. However, these studies implicate new signalling pathways and pave avenues for further optimization and characterization of such novel PSCs (Fig. 2). Mechanistically it will be interesting to test whether there is a connection between RAF and aPKC inhibition and the influence of modulating WNT signalling by applying GSK3i/IWR1 module ${ }^{73}$ on human naïve PSCs. Further, RHO signalling has been shown to promote YAP/TAZ nuclear localization in primed human ESCs and sustain their pluripotency ${ }^{104}$. Thus it remains to be defined whether ROCKi influencing naïve pluripotency characteristics $^{70}$ via YAP/TAZ modulation.

The role of MEK independent FGF2 and TGF $\beta 1 /$ ACTIVIN A signalling, either autocrine or exogenously provided at low doses, remains to be understood in human naïve PSCs. The latter demonstrate upregulation of Activin like ligand GDF $3^{96}$, and human, but not mouse, ICM cells abundantly express Activin receptors ${ }^{97}$. Thus, it is tempting to speculate that primed human ESCs are relatively less primed than murine EpiSC due to differences in response to Activin-like ligands, where it might promote some naïve features in human ${ }^{70,95}$, but not in mouse. To conclude, systematic analysis of the response of pluripotent states from different species to a variety of TGF ligand family members is of importance (Fig. 2), while the possibility to generate human PSCs that are entirely independent of FGF and/or TGF signalling cannot be excluded.

\section{Differences between mouse and human epiblasts}

Recent studies focusing on single cell RNA-seq of human pre-implantation embryos are starting to provide answers to some of the questions highlighted above. While human and mouse blastocyst do not display morphological differences, they retain profound molecular differences at the cellular level ${ }^{97}$ (Fig. 5a,b). Human ICM epiblast cells do not express genes that are considered important pluripotency factors in mouse, such as KLF2 and ESRRB. Instead, KLF17 might have a human-specific role in the $\mathrm{ICM}^{97}$. ERAS, an ESC specific form of RAS, became a pseudo-gene in humans ${ }^{105}$, and Eras null mouse ESCs propagate slowly in FBS/LIF conditions $^{105}$. Non-human primate (Marmoset) pluripotent epiblast is similar transcriptionally to human, and very different than mouse ${ }^{106}$. This includes lack of KLF2, NR0B1 and BMP4 transcription, while acquiring high expression of NODAL and its downstream signalling mediators in human and marmoset naïve $\mathrm{ICM}^{106}$.

At the post implantation stage, major differences exist between rodent and human embryos (Fig. 5a,b). Rodents are rather unusual as their post-implantation epiblast assumes egg-like cylinder shape, while in humans the post-implantation epiblast assumes a flat disc shape, like in most other mammals. While it might be impossible to conduct single cell analysis on early human post-implantation epiblasts, non-human primates might provide some relevant insights. Collectively, these species differences might directly influence the distinct pluripotent characteristics observed in PSCs from different species in vitro and their distinct growth 
requirements. Further, they are of relevance for understanding the developmental context of human in vitro isolated pluripotent cells.

\section{$\underline{\text { A framework for classification of pluripotent states }}$}

The advent of different conditions to isolate human naïve PSCs with distinct characteristics, and the limitations in conducting chimeric analysis in humans, simulate discussions on classifying pluripotent states. It is often claimed that ability to derive ESCs for human ICMs in a newly devised growth condition constitutes "a gold standard" for proving naivety. However, it should be kept in mind that the pluripotent state identity is eventually dictated by the derivation growth condition and not by whether their source was from the pre- or post-implantation epiblast ${ }^{30,33}$, or $\mathrm{PGCs}^{82}$. Utilization of OCT4 distal vs. proximal enhancer element as a binary distinguishing marker can be also misinterpreted ${ }^{95}$. Both OCT4 distal and proximal enhancer elements are active in naïve and primed states, both in humans and mice ${ }^{107,108}$. The difference rather emerges from their relative activity levels (high/low) and dominance.

Relying on a single attribute marker or functional test is limiting and must be accompanied by systematic analysis of ever increasing characteristics that continue to be uncovered for different pluripotent states (Fig. 3,4). Nevertheless, in our opinion, a molecular and functional characteristic that can be considered as a major divider between naïve vs. primed pluripotent states is their response to the challenge of blocking MEK signalling (Fig. 5c). Human conventional ESCs and mouse EpiSCs rapidly collapse upon MEK inhibition, while naïve pluripotent cells rather tolerate and consolidate their naivety following this challenge ${ }^{94}$. Emphasizing this functional quality is supported by the ability of MER/ERK inhibition to expand murine epiblast in ICMs and that it signifies consolidation of naïve pluripotency in vivo ${ }^{109}$.

Within the domains of the naïve and primed ground states of pluripotency, it is clear that if one considers a list of many naïve and primed pluripotency features originally described for mouse naive $2 \mathrm{i} / \mathrm{LIF}$ ESCs and primed FGF2/Activin A EpiSCs, different pluripotency growth conditions can simultaneously endow a mix of primed and naïve properties in the same cell type (Fig. 3). As such, pluripotent states can be classified as "more naïve" or "more primed" by having more of such properties (Fig. 5c). Human primed ESCs have a number of naïve pluripotency features (e.g. protection of promoter regions from hypermethylation, dependence on Nanog), and recent comparative analysis with single cell RNA sequencing of human blastocysts suggests that some conventional human ESC lines maybe transcriptionally relatively less primed than previously thought ${ }^{97}$. Murine naïve ESCs expanded in FBS/LIF conditions can give rise to "all-ESC" chimeric embryos and tolerate Dnmt1/Mettl3 ablation, however they are globally hypermethylated and acquire H3K27me3 over developmental genes as seen in EpiSCs (Fig. 3,5c). Thus, the latter conditions reflect slightly different developmental stages, with FBS/LIF conferring less naïve mouse ESCs than $2 \mathrm{i} /$ LIF conditions.

Other functional tests that can be used to assess the stringency and extent of naivety in different primate naïve PSCs, is whether the cells can tolerate complete ablation of epigenetic repressors like METTL3, DNMT1, DGCR8 and $\operatorname{MBD}^{4,66}$ (Fig. 3,4). Further, such tests might be useful for optimizing conditions that close the gap between mouse and human naive pluripotent cells isolated thus far (Fig. 4, Box 1). Collectively, it will be informative to annotate different naïve and primed sates from any other species isolated thus far according to such criteria (Fig. 3).

\section{Implications and future directions}


Yamanaka's breakthrough of reprogramming somatic cells to pluripotency has provided the foundation for deeper sleuthing of pluripotent states and the understanding that pluripotent configurations can be rewired. The latter, feeds back and bares direct influence on issues associated with current hurdles and limitations related to human iPSC quality and characteristics (Box 1,2).

One of the most fascinating questions related to the naïve to primed pluripotency continuum is "why do these divergent pluripotent configurations actually exist? The latter is often accompanied by the pragmatic question of "Which cells are better to work with - naïve or primed?" In our opinion, as this phenomenon is deeply rooted in early embryonic development in vivo, it is likely that both configurations constitute essential and integral parts for safeguarding optimality and maximizing the benefits of multi-potency and lineage specification simultaneously. We hypothesize that naive pluripotency emerged as an epigenetic erasure state that renders pluripotent cells free from lineage and epigenetic restriction, while simultaneously making these cells relatively less responsive to signalling pathways that might interfere with establishing such a lineage neutral state. The induction of specification by morphogens may not be efficiently enforced during or immediately after establishing naïve pluripotency, without a short "delay period". As such, the naïve pluripotency network is gradually resolved and becomes more receptive to inductive cues at the post-implantation stage, and PSCs get differentially patterned/primed according to their spatial localization, before overt somatic differentiation occurs.

At the functional level, it remains to be established whether using human naïve PSCs as a starting material, with or without a brief priming, would resolve hurdles currently faced in human in vitro PSC differentiation protocols: 1) Will human naïve PSCs yield increased consistency in differentiation among independent iPSC lines ${ }^{110}$ ? 2) Can naïve PSC conditions yield better quality cells in differentiation protocols when used as a starting material? 3) Can human naïve PSC facilitate the success of differentiation protocols that have not been conductive with conventional human PSCs? Encouraging support for the latter has recently been provided by showing the enhanced ability of human PSCs expanded in NHSM conditions (even in the absence of aPKCi) for undergoing in vitro differentiation into PGCs, a protocol that was inefficient from primed human PSCs ${ }^{111}$. The molecular rationale for evaluating the potential benefits highlighted above is that naïve pluripotency is more associated with cleansing of epigenetic repressive marks over regulatory regions, compared to primed cells ${ }^{70,96}$. This might enable more adequate activation of lineage specifiers during differentiation. Further, lineage biases in human primed PSCs are heavily associated with localized accumulation of repressive marks like DNA methylation ${ }^{12}$.

The recent advances in generating human naïve PSCs will continue to boost attempts to generate naïve-like PSCs from other species, and test same-species and inter-species embryo chimerism assays $^{113}$. Cynomolgus monkey naïve ESCs derived in NHSM conditions gave rise to the first chimera competent non-human primate $\mathrm{ESCs}^{114}$. Developmentally advanced mouse embryos (E10.5-E17.5) with low chimerism levels were obtained following injection of naive human ${ }^{70}$ or monkey iPSCs ${ }^{115}$. These observations raise a variety of exciting challenges relating to defining what are the frequency, lineage preference and developmental quality of such integrated primate iPSC derived cells. Systematic efforts will be key to conclude whether humanized animal models ${ }^{70}$ might become relevant for disease modelling, studying human development or generate transplantable human organs ${ }^{113}$.

Continued breakthroughs in single cell technologies and applying them on different pluripotent cell types and embryonic samples will facilitate defining properties that are relevant for adequate 
functionality of PSCs. This will help set standards for optimal starting material for stem cell based therapeutics and research (Box 1). It is expected that during this journey aiming at allowing scientists to better control cell fate by deconstructing this previously underappreciated complexity of pluripotency, proposed criteria and standards will likely be debated and revised. 


\section{Box 1 | A 'dark side’ of naïve pluripotency?}

With the development of naïve conditions and efforts to endow these cells with more features of naivety, one question that emerges is "how much naivety is needed and is there a dark side for permanently maintaining PSCs under naive conditions?"

Rodent ESCs expanded in 2i/LIF have an increased tendency for acquiring genomic abnormalities $^{41}$, and it remains unclear whether these alterations occur as a by-product of nonspecific activity of small molecule inhibitors used ${ }^{95,116}$, or as a direct result of intrinsic molecular features of naïve pluripotency (e.g. increased endogenous retroviral elements (ERVs) activity, reduction in epigenetic repressive marks). One can envision a scenario where such features can be tolerated in vivo where this configuration exists for only 1-2 days, while prolonged in vitro expansion of this state increases the occurrence frequency of such unwanted damaging events.

The latter concern may also relate to safeguarding the integrity of DNA methylation and imprinting in naïve PSCs expanded in vitro over an extended period of time. Studies focusing on loss of DNA methylation upon transfer of mouse PSCs into 2i/LIF conditions have quantified methylation after 10-24 days of transfer, and have documented rapid global loss of DNA methylation accompanied with relative resistance of retrotransposons and imprinting regions to such demethylation ${ }^{83}$. However, it is unclear whether that latter described methylation state represents a final plateau that naive cells achieve, or whether carrying out $2 \mathrm{i} / \mathrm{LIF}$ cultures further eventually leads to erosion in the relative resistance of such regions to demethylation. Indeed, methylation over imprinted genes and retrotransposons are partially, yet significantly, reduced in 2i/LIF conditions ${ }^{83}$.

If such effects are frequent, researchers will have to re-evaluate how to optimize applying naive conditions. One scenario might involve decreasing inhibitor levels as a mean to avoid excessive hypomethylation or other unwanted effects. An alternative scenario is to maintain cells in primed conditions and transfer them into naive conditions only for short time before initiation of differentiation.

\section{Box 2 | Potential implications on iPSC reprogramming.}

Recent studies have implicated how certain epigenetic regulators in fact have opposing effects on naïve and primed murine PSC maintenance (Fig. 4) ${ }^{66}$. These findings might be relevant when comparing induction of pluripotency mechanisms in human vs. mouse, as human, but not mouse iPSCs, are typically reprogrammed in conventional/primed pluripotency conditions ${ }^{117}$. Consequently, some of the differences observed between human and mouse iPSC regulators might not be related to species differences but rather to the fact that distinct pluripotent states are being induced. Therefore, it would be imperative to expand human iPSC reprogramming regulator screens, and include different pluripotency conditions, as they might yield different outcomes.

Another implication of pluripotent state characteristics on reprogramming is whether naïve conditions might improve the quality of obtained iPSCs and facilitate loss of residual epigenetic memory and heterogeneity, when observed ${ }^{118,119}$. Similarly, subtle epigenetic differences in DNA methylation found between NT-ESCs and iPSCs generated from the same human donor cells $^{120}$ might also be neutralized when deriving iPSCs in naïve conditions that mimic more closely epigenetic features of the ICM. 


\section{Display items (all will be subject to graphic editing by NRMCB after review)}

Figure $1 \mid$ Routes for deriving different mouse and human pluripotent stem cell types. A variety of pluripotent cell types can be derived from different embryonic cells harvested at various stages of mouse or human development. Alternatively, somatic cells can be reprogrammed via somatic cell nuclear transfer (SCNT) or in vitro reprogramming via exogenous transcription factors to generate iPSCs. Post-implantation epiblast or germ cell lineage derived pluripotent cells (EpiSCs, EG and GSCs) have not been stably derived yet in human or other primates. For therapeutic purposes, iPSCs and NT-ESCs have the advantage of being genetically identical to donor original somatic cells. However, NT-ESCs will retain mitochondria of the a-nucleated donor female oocyte. Spermatogonial germ stem cells are generated from spermatogonial stem cells that have already established exclusive male/paternal imprinting pattern, and so do GSCs derived from them. If established from adult human males in the future, such maternal imprint-free characteristics will likely limit future therapeutic potential of human GSCs.

Figure 2 | Signalling pathways and their influence on naïve and primed pluripotent states. Figure delineates different signalling pathways and their ability to positively or negatively regulate naïve and primed murine pluripotent cells. Please note how that the majority of signalling pathways delineated have opposing effects on murine naïve vs. primed pluripotent state stability (e.g. Lif/Stat3, Fgf2/Erk). It is important to highlight that other pathways not included in this scheme, are likely to be involved in such regulation and will likely be further characterized over the next years. This may include Hippo, Rho, Notch and NFkB signalling. Purple dotted boxes highlight signalling pathways that may be acting differently in mouse vs. human PSC regulation. More specifically, it remains to be fully understood whether low dose of TGF $\beta$, ACTIVIN/NODAL, nuclear $\beta$ Catenin or FGF2 (MEK/ERK independent) signalling influence human naïve pluripotency differently than previously observed in rodent naïve ESCs. Dotted arrow indicates potential links that remain to be established. (Image is reused and modified from our Nature Reviews Molecular Cell Biology Poster (http://www.nature.com/nrm/posters/pluripotency/index.html)).

Figure 3 | Naïve and primed pluripotent cell properties in different isolated PSCs.

The scheme delineates in the first column on the left a list of different properties that distinguish between murine ESCs expanded in 2i/LIF (naïve) and murine EpiSCs expanded in Fgf2/Activin A (primed). The latter two states are used as a reference to annotate a variety of other naïve and primed conditions devised for mouse, rat, human or monkey PSCs. For each condition and for each stem cell property, we indicate whether it retains naïve-like (orange circle) or primed-like pattern (blue circle). Empty boxes indicate lack of characterization. This list of features is likely to expand with time and can be used to systematically annotate new pluripotent states isolated in unique conditions and from different species. (Image is reused and modified from our Nature $\begin{array}{llll}\text { Reviews } & \text { Molecular } & \text { Cell } & \text { Poster }\end{array}$ (http://www.nature.com/nrm/posters/pluripotency/index.html)).

\section{Figure 4 | Opposing influence for epigenetic repressors on murine naïve and primed} pluripotent cells.

Murine naïve and primed pluripotent cells do not only differ in their dependence on distinct signalling pathways or in their epigenetic profile, but also in their lineage decision-making. Murine naïve ESCs expanded either in 2i/LIF and FBS/LIF conditions, tolerate complete loss of epigenetic repressors such as Dnmt1, Mbd3, Dicer, Dgcr8, Mettl3, Eed, Ezh2. Further, the latter modifications strengthen the equilibrium in favour of pluripotency promoting factors and generates "hyper-naïve" pluripotent cells that are relatively more resistant to differentiation and 
can tolerate withdrawal of LIF cytokine. Primed EpiSCs generally respond in an opposite manner to the complete ablation of such repressors. Established murine primed EpiSCs naturally down regulate pluripotency factors and upregulate lineage priming factors. Ablation of repressors at this stage tips the balance toward differentiation and/or compromises cell survival. It should be noted that ablation of different repressors (e.g. Mettl3 vs. Dgcr8) overall negatively influences primed pluripotency stability, this does not necessarily mean that the downstream events leading to the state collapse are identical, and thus should be thoroughly dissected in vitro and in vivo.

Figure 5 | Key differences between mouse and human pre and post-implantation development in vivo. (a) Scheme delineates similarities and differences in mouse and human early pre- and post-implantation development. While mouse and human embryos are not morphologically different up to the blastocyst stage, there are striking transcriptional differences as summarized in (b). Further, at the postimplantation stage, morphological differences in human vs. mouse embryo shape become striking including (i) differences in epiblast shape and (ii) extra-embryonic structures, as delineated in (a). In mouse, naïve ESCs (orange arrows) can be derived from ICM or post-implantation epiblast when naïve growth conditions are applied. Primed EpiSCs can be derived from post-implantation epiblast or ICM when primed culture conditions are used. Thus, growth condition rather than source dictates pluripotent state configuration acquired in vitro. A similar scenario applies for derivation of naïve and primed cells from human ICM depending on the growth conditions used. PSC derivations or molecular analysis on human post-implantation embryos cannot be conducted due to ethical issues. PEPrimitive Endoderm; TE - Trophectoderm; ICM- Inner Cell Mass. (c) A model to explain the relativity of naivety within the naive to primed pluripotency spectrum. One major molecular and functional criterion that can be considered for separating naïve and primed pluripotent cells is their ability to maintain and stabilize their pluripotent state upon induced blocking of MEK activity (dashed black line). Within the naïve or primed pluripotent states, it is difficult to describe the pluripotent state of the cells in absolute terms, as naïve cells can have, to some extent, primed pluripotency features. Similarly, within the primed pluripotency spectrum, primed PSCs expanded in different conditions have different features and varying degrees of naivety (Figure 3). Finally, it is possible that supplementation of 2i/LIF conditions with small molecules such as aPKCi, FGFRi or NOTCHi will further consolidate naïve pluripotency features, particularly from other rodents like rats whose stability in $2 \mathrm{i} /$ LIF feeder free conditions should be further improved. Full annotation of different human pluripotent states will allow charting an equivalent landscape for human and monkey PSCs. (Images in a-b are reused and modified from our Nature Reviews Molecular Cell Biology Poster (http://www.nature.com/nrm/posters/pluripotency/index.html). Image in $\mathbf{c}$ is reused and modified from our Figure 3 in Manor et al. Curr. Opin. in Genetics \& Development).

\section{Copyright permissions}

- Figure 2,3,5a-b: Graphic figures are reused from our Nature Reviews Molecular Cell Biology Poster

(http://www.nature.com/nrm/posters/pluripotency/index.html) as indicated in figure legends.

- Figure $5 \mathrm{c}$ is reused and modified from our Figure 3 in Manor et al. Curr. Opin. In Genetics \& development 


\section{Acknowledgements}

J.H.H is supported by a generous gift from Ilana and Pascal Mantoux; the New York Stem Cell Foundation (NYSCF), Flight Attendant Medical Research Institute (FAMRI), the Kimmel Innovator Research Award, the ERC-StG (StG-2011-281906) and ERC-PoC (PoC-2015692945), Moross Cancer Institute, Israel Science Foundation - NFSC program, Morasha Biomed program, ICORE program, the ICRF Foundation, MINERVA fund, Helen and Martin Kimmel Institute for Stem Cell research, the Benoziyo Endowment fund, David and Fela Shapell Family Foundation INCPM Fund for Preclinical Studies, an HFSPO research grant. J.H.H. is a New York Stem Cell Foundation - Robertson Investigator. We thank W. Greenleaf and members of the Hanna lab for discussions. We apologize to those whose work could not be covered or directly cited due to space limitations.

\section{Further information}

Online Web link sites:

Addgene plasmid repository: https://www.addgene.org/

ENCODE project: http://www.nature.com/encode/

Epigenome Roadmap project: http://www.nature.com/collections/vbqgtr

Mouse ES cell ChIP compendium: http://bioinformatics.cscr.cam.ac.uk/ES_Cell_ChIPseq compendium.html

Mouse ES Single cell RNA-seq resource - ESpresso: http://www.ebi.ac.uk/teichmann$\underline{\text { srv/espresso/ }}$

CRISPR/CAS9 genome wide screen resource: $\underline{\text { http://genome-engineering.org/gecko/ }}$

Online poster by Nature Reviews Molecular Cell Biology on Pluripotent States:

http://www.nature.com/nrm/posters/pluripotency/index.html 


\section{Glossary}

Primordial germ cells (PGCs) - embryonic progenitor cells that give rise to germ cells in the gonads (sperm and oocytes).

Inner cell mass (ICM) - the mass of cells inside the pre-implantation blastocyst that will subsequently give rise to the definitive structures of the fetus.

Embryonic stem cells (ESCs) - in vitro expanded pluripotent cells that originate from the ICM.

Epiblast stem cells (EpiSCs)- in vitro expanded pluripotent cells that originate from the postimplantation epiblast.

Embryonic germ cells - in vitro expanded pluripotent cells that are derived from embryonic PGCs.

Germ stem cells (GSCs) - in vitro expanded pluripotent stem cells that originate from neonatal or adult testis derived spermatogonial stem cells.

Nuclear transfer - cloning of somatic cell derived nucleus and its introduction into a-nucleated host oocyte.

Induced pluripotent stem cells (iPSCs) - in vitro generated pluripotent cells derived via ectopic expression of defined exogenous factors in somatic cells.

Naïve pluripotency - pluripotent state that resembles pre-implantation pluripotent configuration(s).

Primed pluripotency - pluripotent state that resembles to post-implantation embryonic configuration(s).

Ground state pluripotency - originally described as a state of pluripotency that is independent of exogenous activator signalling input or stimulation.

$\mathrm{X}$ inactivation - dosage compensation of $\mathrm{X}$ chromosome in female, where one of the $\mathrm{X}$ chromosomes gets epigenetically silenced.

Seed enhancers - subgroup of enhancers that are dormant in naive cells but become more active in primed pluripotent and somatic cells.

$3 \mathrm{i}$ - Defined naïve pluripotency growth conditions combing 3 inhibitors (i) for MEK, FGF and GSK3 signalling.

2i/LIF - Defined naïve pluripotency growth conditions containing 2 inhibitors (i) for MEK and GSK3 together with LIF cytokine.

"Alternative 2i" - Defined naïve pluripotency growth conditions composed of 2 small molecule inhibitors for GSK3 and SRC pathways

LIF/MEKi/aPKCi - Defined naïve pluripotency growth conditions containing 2 inhibitors (i) for MEK and atypical PKC signalling, together with LIF cytokine. 
FGF2/ACTIVIN A - Defined primed pluripotency growth conditions for mouse EpiSCs composed of recombinant FGF2 and ACTIVIN A cytokines.

GSK3i/IWR1 - Defined primed pluripotency growth conditions for mouse EpiSCs composed of GSK3 pathway inhibitor and Tankyrase small molecule inhibitor, IWR1.

FGF2/IWR1 - Defined primed pluripotency growth conditions for mouse EpiSCs composed of recombinant FGF2 and Tankyrase small molecule inhibitor, IWR1.

\section{$\underline{\text { Table of contents summary }}$}

Hanna and colleagues review recent advances on molecular underpinnings of alternative primedand naïve-like pluripotent states isolated in rodents and in man. They highlight potential benefits and identify key unanswered challenges in this rapidly evolving fundamental topic.

\section{Online summary}

1. Pluripotency is highly dynamic in vivo and evolves at different stages of pre- and postimplantation stages. However, the self-renewal aspect is a highly useful in vitro artificial phenotype endowed by culture conditions.

2. Different pluripotent cell types can be isolated in vitro from different sources and methods. The Pluripotent state assumed by the cultivated cells is dictated by the in vitro growth condition, rather than their cell of origin.

3. Naïve and primed pluripotent states can be functionally classified based on their ability or failure to maintain self-renewal of their pluripotent state upon MEK signaling inhibition, respectively.

4. Naïve and primed states of pluripotency can each represent a continuum of configurations rather than a fixed individual state. Within the naïve and primed pluripotent states, different degrees of naiveté or priming can be captured and annotated based on a variety of characteristics.

5. Human conventional pluripotent cells are primed, however they are not identical to mouse primed cells, and have certain naïve-like properties. In vivo differences likely underlie differences in growth requirements and characteristics of pluripotent cells isolated in vitro from mice and man.

6. The use of human naïve pluripotent growth conditions and cells might have great influence on $\mathrm{iPSC} / \mathrm{ESC}$ quality and their differentiation competence, consistency and robustness. 


\section{$\underline{\text { Author biographies }}$}

Leehee Weinberger M.Sc., has been a PhD student with Jacob H. Hanna at the Department of Molecular Genetics at the Weizmann Institute of Science, Rehovot, Israel for 4 years. She carried out her Masters work with Naama Barkai at the the same department. Her doctoral research focused on regulation and differentiation of human naïve pluripotent cells and germ cells.

Muneef Ayyash Ph.D., has been a staff researcher in Jacob H. Hanna lab at the Department of Molecular Genetics at the Weizmann Institute of Science Rehovot, Israel for 1 year. He carried his doctoral work with David Wallach at the same institute and did postdoctoral studies with Yinon Ben-Neriah at the Hebrew University, Jerusalem, Israel. His research currently focuses on WNT and HIPPO signaling modulation in promoting human naïve pluripotency.

Noa Novershtern Ph.D., has been a staff scientist and senior bioinformatician in Jacob H. Hanna lab at the Department of Molecular Genetics at the Weizmann Institute of Science, Rehovot, Israel for 5 years. She carried out her doctoral work with Nir Friedman at the Hebrew University, Jerusalem, Israel and Aviv Regev at the Broad Institute, Cambridge, USA. She focuses on dissecting epigenetic dynamics in pluripotency and reprogramming.

Jacob H. Hanna M.D. Ph.D., has been on the Faculty of the Department of Molecular Genetics at the Weizmann Institute of Science, Rehovot, Israel for 5 years, and is also a Robertson Investigator by the New York Stem Cell Foundation (NYSCF). He carried out his doctoral work with Ofer Mandelboim at the Hebrew University, Jerusalem, Israel and then did his postdoctoral studies with Rudolf Jaenisch at the Whitehead Institute for Biomedical Research - Massachusetts Institute of Technology, Cambridge, USA. His laboratory studies molecular mechanisms of stem cell reprogramming and differentiation. Hanna lab website: http://hannalabweb.weizmann.ac.il/ 


\section{$\underline{\text { References }}$}

1 Hanna, J. H., Saha, K. \& Jaenisch, R. Pluripotency and cellular reprogramming: facts, hypotheses, unresolved issues. Cell 143, 508-525 (2010).

2 Nichols, J. \& Smith, A. Pluripotency in the embryo and in culture. Cold Spring Harb Perspect Biol 4, a008128 (2012).

3 Hackett, J. A. \& Surani, M. A. Regulatory principles of pluripotency: from the ground state up. Cell Stem Cell 15, 416-430 (2014).

4 Manor, Y. S., Massarwa, R. \& Hanna, J. H. Establishing the human naive pluripotent state. Curr Opin Genet Dev 34, 35-45 (2015).

5 Inoue, H., Nagata, N., Kurokawa, H. \& Yamanaka, S. iPS cells: a game changer for future medicine. EMBO J 33, 409-417 (2014).

**6 Thomson, J. A. et al. Embryonic stem cell lines derived from human blastocysts. Science 282, 1145-1147 (1998).

First study to derive primed ESCs from human blastocysts.

7 Evans, M. J. \& Kaufman, M. H. Establishment in culture of pluripotential cells from mouse embryos. Nature 292, 154-156 (1981).

8 Martin, G. R. Isolation of a pluripotent cell line from early mouse embryos cultured in medium conditioned by teratocarcinoma stem cells. Proc Natl Acad Sci U S A 78, 76347638 (1981).

**9 Tesar, P. J. et al. New cell lines from mouse epiblast share defining features with human embryonic stem cells. Nature 448, 196-199, doi:10.1038/nature05972 (2007). One of two studies that derived primed EpiSC lines from rodent post-implantation epiblasts.

**10 Brons, I. G. et al. Derivation of pluripotent epiblast stem cells from mammalian embryos. Nature 448, 191-195 (2007).

One of two studies that derived primed EpiSC lines from rodent post-implantation epiblasts.

11 Leitch, H. G. et al. Embryonic germ cells from mice and rats exhibit properties consistent with a generic pluripotent ground state. Development 137, 2279-2287 (2010).

*12 Y, M., K, Z. \& Bl, H. Derivation of pluripotential embryonic stem cells from murine primordial germ cells in culture. Cell 70, 841-847 (1992).

First study to desribe generation of pluripotent ES-like cells from mouse embryonic primordial germ cells.

*13 Kanatsu-Shinohara, M. et al. Generation of pluripotent stem cells from neonatal mouse testis. Cell 119, 1001-1012 (2004).

First study to desribe generation of pluripotent ES-like cells from mouse spermatogonial stem cells. 
14 Tanaka, T., Kanatsu-Shinohara, M., Hirose, M., Ogura, A. \& Shinohara, T. Pluripotent cell derivation from male germline cells by suppression of Dmrt1 and Trp53. J Reprod Dev 61, 473-84 (2015).

15 Ko, K. et al. Induction of pluripotency in adult unipotent germline stem cells. Cell Stem Cell 5, 87-96 (2009).

16 Ko, K. et al. Human adult germline stem cells in question. Nature 465, E1; discussion E3 (2010).

17 Shamblott, M. J. et al. Derivation of pluripotent stem cells from cultured human primordial germ cells. Proc Natl Acad Sci U S A 95, 13726-13731 (1998).

18 Yamada, M. et al. Human oocytes reprogram adult somatic nuclei of a type 1 diabetic to diploid pluripotent stem cells. Nature 510, 533-536 (2014).

**19 Tachibana, M. et al. Human embryonic stem cells derived by somatic cell nuclear transfer. Cell 153, 1228-1238 (2013).

First study to generate validated human NT-ESCs from somatic fibroblasts.

*20 Wakayama, T. et al. Differentiation of embryonic stem cell lines generated from adult somatic cells by nuclear transfer. Science 292, 740-743 (2001).

Study demonstrating feasibility of SCNT in mice.

**21 Takahashi, K. \& Yamanaka, S. Induction of pluripotent stem cells from mouse embryonic and adult fibroblast cultures by defined factors. Cell 126, 663-676 (2006).

First study that invented direct in vitro reprogramming of somati cells into iPSCs with defined transcription factors.

22 Takahashi, K. et al. Induction of pluripotent stem cells from adult human fibroblasts by defined factors. Cell 131, 861-872 (2007).

23 Wernig, M. et al. In vitro reprogramming of fibroblasts into a pluripotent ES-cell-like state. Nature 448, 318-324 (2007).

$24 \mathrm{Yu}$, J. et al. Human induced pluripotent stem cells free of vector and transgene sequences. Science 324, 797-801 (2009).

$25 \mathrm{Yu}$, J. et al. Induced pluripotent stem cell lines derived from human somatic cells. Science 318, 1917-1920 (2007).

26 Deuse, T. et al. SCNT-derived ESCs with mismatched mitochondria trigger an immune response in allogeneic hosts. Cell Stem Cell 16, 33-38 (2015).

27 Ma, H. et al. Metabolic rescue in pluripotent cells from patients with mtDNA disease. Nature 524, 234-238 (2015).

28 Tachibana, M. et al. Mitochondrial gene replacement in primate offspring and embryonic stem cells. Nature 461, 367-372 (2009). 
29 Paull, D. et al. Nuclear genome transfer in human oocytes eliminates mitochondrial DNA variants. Nature 493, 632-637 (2013).

**30 Hanna, J. et al. Metastable pluripotent states in NOD-mouse-derived ESCs. Cell Stem Cell 4, 513-524 (2009).

One of two studies first showing interconversion between murine naïve and primed pluripotent cells, and first to define distinct requirements in different mouse strains for in vitro and ex vivo interconversions.

31 Nichols, J. \& Smith, A. Naive and primed pluripotent states. Cell Stem Cell 4, 487-492 (2009).

32 Han, D. W. et al. Direct reprogramming of fibroblasts into epiblast stem cells. Nat Cell Biol 13, 66-71 (2011).

33 Najm, F. J. et al. Isolation of epiblast stem cells from preimplantation mouse embryos. Cell Stem Cell 8, 318-325 (2011).

34 Martin, G.R. \& Evans, M.J. Differentiation of clonal lines of teratocarcinoma cells: formation of embryoid bodies in vitro. Proc Natl Acad Sci U S A 72, 1441-1445 (1975).

35 Smith, A. G. et al. Inhibition of pluripotential embryonic stem cell differentiation by purified polypeptides. Nature 336, 688-690 (1988).

36 Williams, R. L. et al. Myeloid leukaemia inhibitory factor maintains the developmental potential of embryonic stem cells. Nature 336, 684-687 (1988).

37 Orkin, S. H. \& Hochedlinger, K. Chromatin connections to pluripotency and cellular reprogramming. Cell 145, 835-850 (2011).

38 Ying, Q. L., Nichols, J., Chambers, I. \& Smith, A. BMP induction of Id proteins suppresses differentiation and sustains embryonic stem cell self-renewal in collaboration with STAT3. Cell 115, 281-292 (2003).

39 Burdon, T., Stracey, C., Chambers, I., Nichols, J. \& Smith, A. Suppression of SHP-2 and ERK signalling promotes self-renewal of mouse embryonic stem cells. Dev Biol 210, 30 43 (1999).

**40 Ying, Q. L. et al. The ground state of embryonic stem cell self-renewal. Nature 453, 519523 (2008).

Study describing defined 3i nä̈ve conditions capable of generating Lif/Stat3 independent mouse ESCs.

41 Buehr, M. et al. Capture of authentic embryonic stem cells from rat blastocysts. Cell 135, 1287-1298 (2008).

42 Nichols, J. et al. Validated germline-competent embryonic stem cell lines from nonobese diabetic mice. Nat Med 15, 814-818 (2009).

43 Chen, H. et al. Erk signaling is indispensable for genomic stability and self-renewal of mouse embryonic stem cells. Proc Natl Acad Sci U S A 112, E5936-5943 (2015). 
44 Shimizu, T. et al. Dual inhibition of Src and GSK3 maintains mouse embryonic stem cells, whose differentiation is mechanically regulated by Src signaling. Stem Cells $\mathbf{3 0}$, 1394-1404 (2012).

*45 Dutta, D. et al. Self-renewal versus lineage commitment of embryonic stem cells: protein kinase C signaling shifts the balance. Stem Cells 29, 618-628 (2011).

First study utilizing aPKCi to boost naïve murine iPSCs and ESCs generation.

46 Kolodziejczyk, A. A. et al. Single Cell RNA-Sequencing of Pluripotent States Unlocks Modular Transcriptional Variation. Cell Stem Cell 17, 471-485 (2015).

47 Chen, Y., Blair, K. \& Smith, A. Robust self-renewal of rat embryonic stem cells requires fine-tuning of glycogen synthase kinase-3 inhibition. Stem Cell Reports 1, 209-217 (2013).

48 Meek, S. et al. Tuning of beta-catenin activity is required to stabilize self-renewal of rat embryonic stem cells. Stem Cells 31, 2104-2115 (2013).

49 Rajendran, G. et al. Inhibition of protein kinase C signaling maintains rat embryonic stem cell pluripotency. J Biol Chem 288, 24351-24362 (2013).

*50 Li, X. et al. Calcineurin-NFAT signaling critically regulates early lineage specification in mouse embryonic stem cells and embryos. Cell Stem Cell 8, 46-58 (2011).

First study to show that Src inhibition promotes murine naïve pluripotency.

**51 Nishioka, N. et al. The Hippo signaling pathway components Lats and Yap pattern Tead4 activity to distinguish mouse trophectoderm from inner cell mass. Dev Cell 16, 398-410 (2009).

Study identifies the role of Hippo signaling pathway in epiblast vs. trophoblast specification in pre-implantaioon mouse embryos.

52 Azzolin, L. et al. YAP/TAZ incorporation in the beta-catenin destruction complex orchestrates the Wnt response. Cell 158, 157-170 (2014).

53 Lian, I. et al. The role of YAP transcription coactivator in regulating stem cell selfrenewal and differentiation. Genes Dev 24, 1106-1118 (2010).

54 Wray, J. et al. Inhibition of glycogen synthase kinase-3 alleviates Tcf3 repression of the pluripotency network and increases embryonic stem cell resistance to differentiation. Nat Cell Biol 13, 838-845 (2011).

55 Faunes, F. et al. A membrane-associated beta-catenin/Oct4 complex correlates with ground-state pluripotency in mouse embryonic stem cells. Development 140, 1171-1183 (2013).

56 Morgani, S. M. \& Brickman, J. M. LIF supports primitive endoderm expansion during pre-implantation development. Development 142, 3488-3499 (2015).

57 Silva, J. et al. Nanog is the gateway to the pluripotent ground state. Cell 138, 722-737 (2009). 
58 Maza, I. et al. Transient acquisition of pluripotency during somatic cell transdifferentiation with iPSC reprogramming factors. Nat Biotechnol 33, 769-774 (2015).

59 Carter, A. C., Davis-Dusenbery, B. N., Koszka, K., Ichida, J. K. \& Eggan, K. Nanogindependent reprogramming to iPSCs with canonical factors. Stem Cell Reports 2, 119126 (2014).

60 Schwartz, B.A. et al. Ba, S., O, B.-N., Jc, S. \& K, H. Nanog is dispensable for the generation of induced pluripotent stem cells. Curr Biol 24, 347-350 (2014).

61 Festuccia, N. et al. Esrrb is a direct Nanog target gene that can substitute for Nanog function in pluripotent cells. Cell Stem Cell 11, 477-490 (2012).

62 Yeo, J. C. et al. Klf2 is an essential factor that sustains ground state pluripotency. Cell Stem Cell 14, 864-872 (2014).

**63 Reynolds, N. et al. NuRD suppresses pluripotency gene expression to promote transcriptional heterogeneity and lineage commitment. Cell Stem Cell 10, 583-594 (2012).

Study establishes $\mathrm{Mbd} 3 / \mathrm{NuRD}$ as a repressor of naïve pluriptoency promoting genes in mouse ESCs.

64 Rais, Y. et al. Deterministic direct reprogramming of somatic cells to pluripotency. Nature 502, 65-70 (2013).

65 Loh, K. M. \& Lim, B. A precarious balance: pluripotency factors as lineage specifiers. Cell Stem Cell 8, 363-369 (2011).

*66 Geula, S. et al. Stem cells. m6A mRNA methylation facilitates resolution of naive pluripotency toward differentiation. Science 347, 1002-1006 (2015).

First study proving opposing dependence on epigentic repressors between mouse naïve and primed PSCs.

67 Huang, Y. et al. In Vivo differentiation potential of epiblast stem cells revealed by chimeric embryo formation. Cell Rep 2, 1571-1578 (2012).

68 Buecker, C. et al. Reorganization of enhancer patterns in transition from naive to primed pluripotency. Cell Stem Cell 14, 838-853 (2014).

69 Marks, H. et al. The transcriptional and epigenomic foundations of ground state pluripotency. Cell 149, 590-604 (2012).

**70 Gafni, O. et al. Derivation of novel human ground state naive pluripotent stem cells. Nature 504, 282-286 (2013).

First study to generate geneticaly unmodified and indefinitely stable human MEK independent naive PSCs, also capable of generating advanced cross species mousehuman chimeric embryos. 
71 Factor, D. C. et al. Epigenomic comparison reveals activation of "seed" enhancers during transition from naive to primed pluripotency. Cell Stem Cell 14, 854-863 (2014).

72 Kumar, R. M. et al. Deconstructing transcriptional heterogeneity in pluripotent stem cells. Nature 516, 56-61 (2014).

73 Kim, H. et al. Modulation of beta-catenin function maintains mouse epiblast stem cell and human embryonic stem cell self-renewal. Nat Commun 4, 2403 (2013).

*74 Kojima, Y. et al. The transcriptional and functional properties of mouse epiblast stem cells resemble the anterior primitive streak. Cell Stem Cell 14, 107-120 (2014). One of first two studies describing region-specific features of in vitro expanded mouse EpiSCs.

*75 Wu, J. et al. An alternative pluripotent state confers interspecies chimaeric competency. Nature 521, 316-321 (2015).

One of first two studies describing region-specific features of in vitro expanded mouse EpiSCs.

76 Han, D. W. et al. Epiblast stem cell subpopulations represent mouse embryos of distinct pregastrulation stages. Cell 143, 617-627 (2010).

77 Hayashi, K., Ohta, H., Kurimoto, K., Aramaki, S. \& Saitou, M. Reconstitution of the mouse germ cell specification pathway in culture by pluripotent stem cells. Cell 146, 519-532 (2011).

**78 Guo, G. et al. Klf4 reverts developmentally programmed restriction of ground state pluripotency. Development 136, 1063-1069 (2009).

One of two studies first showing interconversion between murine naïve and primed pluripotent cells.

79 Bao, S. et al. Epigenetic reversion of post-implantation epiblast to pluripotent embryonic stem cells. Nature 461, 1292-1295 (2009).

80 Gillich, A. et al. Epiblast stem cell-based system reveals reprogramming synergy of germline factors. Cell Stem Cell 10, 425-439 (2012).

81 Greber, B. et al. Conserved and divergent roles of FGF signaling in mouse epiblast stem cells and human embryonic stem cells. Cell Stem Cell 6, 215-226 (2010).

82 Leitch, H. G. et al. Naive pluripotency is associated with global DNA hypomethylation. Nat Struct Mol Biol 20, 311-316 (2013).

83 Ficz, G. et al. FGF signaling inhibition in ESCs drives rapid genome-wide demethylation to the epigenetic ground state of pluripotency. Cell Stem Cell 13, 351-359 (2013).

84 Hackett, J. A. et al. Synergistic mechanisms of DNA demethylation during transition to ground-state pluripotency. Stem Cell Reports 1, 518-531 (2013). 
85 Galonska, C., Ziller, M. J., Karnik, R. \& Meissner, A. Ground State Conditions Induce Rapid Reorganization of Core Pluripotency Factor Binding before Global Epigenetic Reprogramming. Cell Stem Cell 17, 462-470 (2015).

86 Liao, J. et al. Targeted disruption of DNMT1, DNMT3A and DNMT3B in human embryonic stem cells. Nat Genet 47, 469-478 (2015).

87 Tee, W. W., Shen, S. S., Oksuz, O., Narendra, V. \& Reinberg, D. Erk1/2 activity promotes chromatin features and RNAPII phosphorylation at developmental promoters in mouse ESCs. Cell 156, 678-690 (2014).

88 Bertero, A. et al. Activin/nodal signaling and NANOG orchestrate human embryonic stem cell fate decisions by controlling the H3K4me3 chromatin mark. Genes Dev 29, 702-717 (2015).

89 Smith, Z. D. et al. DNA methylation dynamics of the human preimplantation embryo. Nature 511, 611-615 (2014).

90 Mekhoubad, S. et al. Erosion of dosage compensation impacts human iPSC disease modeling. Cell Stem Cell 10, 595-609 (2012).

91 Chia, N. Y. et al. A genome-wide RNAi screen reveals determinants of human embryonic stem cell identity. Nature 468, 316-320 (2010).

92 Shipony, Z. et al. Dynamic and static maintenance of epigenetic memory in pluripotent and somatic cells. Nature 513, 115-119 (2014).

93 Betschinger, J. et al. Exit from pluripotency is gated by intracellular redistribution of the bHLH transcription factor Tfe3. Cell 153, 335-347 (2013).

*94 Hanna, J. et al. Human embryonic stem cells with biological and epigenetic characteristics similar to those of mouse ESCs. Proc Natl Acad Sci U S A 107, 9222-9227 (2010).

Study provided first evidence for alternative transgene dependent human PSCs that can be expanded in conditions containing $2 \mathrm{i} / \mathrm{LIF}$.

95 Theunissen, T. W. et al. Systematic identification of culture conditions for induction and maintenance of naive human pluripotency. Cell Stem Cell 15, 471-487 (2014).

96 Takashima, Y. et al. Resetting transcription factor control circuitry toward ground-state pluripotency in human. Cell 158, 1254-1269 (2014).

97 Blakeley, P. et al. Defining the three cell lineages of the human blastocyst by single-cell RNA-seq. Development 142, 3151-3165 (2015).

98 Gkountela, S. et al. DNA Demethylation Dynamics in the Human Prenatal Germline. Cell 161, 1425-1436 (2015).

99 Shakiba, N. et al. CD24 tracks divergent pluripotent states in mouse and human cells. Nat Commun 6, 7329 (2015). 
100 Barakat, T. S. et al. Stable X chromosome reactivation in female human induced pluripotent stem cells. Stem Cell Reports 4, 199-208 (2015).

101 Chan, Y. S. et al. Induction of a human pluripotent state with distinct regulatory circuitry that resembles preimplantation epiblast. Cell Stem Cell 13, 663-675 (2013).

102 Duggal, G. et al. Alternative Routes to Induce Naive Pluripotency in Human Embryonic Stem Cells. Stem Cells 33, 2686-2698 (2015).

103 Chen, H. et al. Reinforcement of STAT3 activity reprogrammes human embryonic stem cells to naive-like pluripotency. Nat Commun 6, 7095 (2015).

*104 Ohgushi, M., Minaguchi, M. \& Sasai, Y. Rho-Signaling-Directed YAP/TAZ Activity Underlies the Long-Term Survival and Expansion of Human Embryonic Stem Cells. Cell Stem Cell (2015).

Study connecting RHO and HIPPO signalling pathways in maintenance of primed human pluripotency.

105 Kameda, T. \& Thomson, J. A. Human ERas gene has an upstream premature polyadenylation signal that results in a truncated, noncoding transcript. Stem Cells 23, 1535-1540 (2005).

106 Boroviak, T. et al. Lineage-Specific Profiling Delineates the Emergence and Progression of Naive Pluripotency in Mammalian Embryogenesis. Dev Cell 35, 366-382 (2015).

107 Buecker, C. \& Wysocka, J. Enhancers as information integration hubs in development: lessons from genomics. Trends Genet 28, 276-284 (2012).

108 Karwacki-Neisius, V. et al. Reduced Oct4 expression directs a robust pluripotent state with distinct signaling activity and increased enhancer occupancy by Oct 4 and Nanog. Cell Stem Cell 12, 531-545 (2013).

109 Boroviak, T., Loos, R., Bertone, P., Smith, A. \& Nichols, J. The ability of inner-cell-mass cells to self-renew as embryonic stem cells is acquired following epiblast specification. Nat Cell Biol 16, 516-528 (2014).

110 Osafune, K. et al. Marked differences in differentiation propensity among human embryonic stem cell lines. Nat Biotechnol 26, 313-315, doi:10.1038/nbt1383 (2008).

**111 Irie, N. et al. SOX17 is a critical specifier of human primordial germ cell fate. Cell 160, 253-268 (2015).

First study to generate human in vitro PGCLCs and demonstrate altered function of human MEK independent naïve PSCs.

112 Bock, C. et al. Reference Maps of human ES and iPS cell variation enable highthroughput characterization of pluripotent cell lines. Cell 144, 439-452 (2011).

113 Kobayashi, T. et al. Generation of rat pancreas in mouse by interspecific blastocyst injection of pluripotent stem cells. Cell 142, 787-799 (2010).

First study to generate cross-species chimerisim between mice and rats via microinjecting naïve PSCs from one species into host blastocysts from the other. 
**114 Chen, Y. et al. Generation of Cynomolgus Monkey Chimeric Fetuses using Embryonic Stem Cells. Cell Stem Cell 17, 116-124 (2015).

Study demonstrating frist ever generated chimeric monkey fetuses with naïve monkey ESCs established in NHSM conditions supplemented with Vitamin C.

115 Fang, R. et al. Generation of naive induced pluripotent stem cells from rhesus monkey fibroblasts. Cell Stem Cell 15, 488-496 (2014).

116 Bock, A. S., Leigh, N. D. \& Bryda, E. C. Effect of Gsk3 inhibitor CHIR99021 on aneuploidy levels in rat embryonic stem cells. In Vitro Cell Dev Biol Anim 50, 572-579 (2014).

117 Qin, H. et al. Systematic identification of barriers to human iPSC generation. Cell 158, 449-461 (2014).

118 Kim, K. et al. Donor cell type can influence the epigenome and differentiation potential of human induced pluripotent stem cells. Nat Biotechnol 29, 1117-1119 (2011).

119 Choi, J. et al. A comparison of genetically matched cell lines reveals the equivalence of human iPSCs and ESCs. Nat Biotechnol 33, 1173-1181 (2015).

120 Ma, H. et al. Abnormalities in human pluripotent cells due to reprogramming mechanisms. Nature 511, 177-183 (2014). 
in vitro established

Human

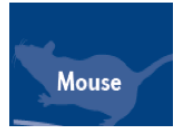

Yes

Yes

No

Yes

No

Yes

Embryonic germ stem cell EG

Male imprinting pattern only

Non-isogenic mitochondria

in vitro

Isolated Pluripotent

Cell Type

Embryonic stem cell

ESC

Epiblast stem cell EpisC

No

Yes

Spermatogonial germ stem cell

Yes Yes

Yes Yes

$$
\begin{gathered}
\text { GSC } \\
\text { Induced pluripotent cell } \\
\text { iPSC }
\end{gathered}
$$

Nuclear Transfer ESC
Preimplantation embryo
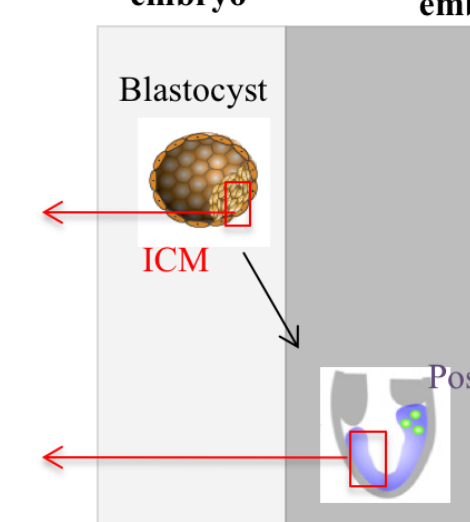

Post-implantation epiblast implantation embryo

\section{Postnatal/adult}

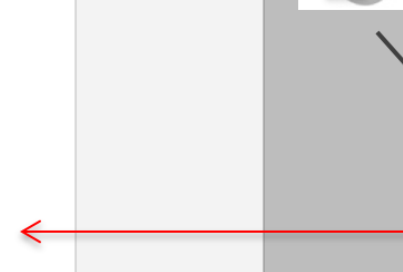

(1)

in vivo

Postmbryo

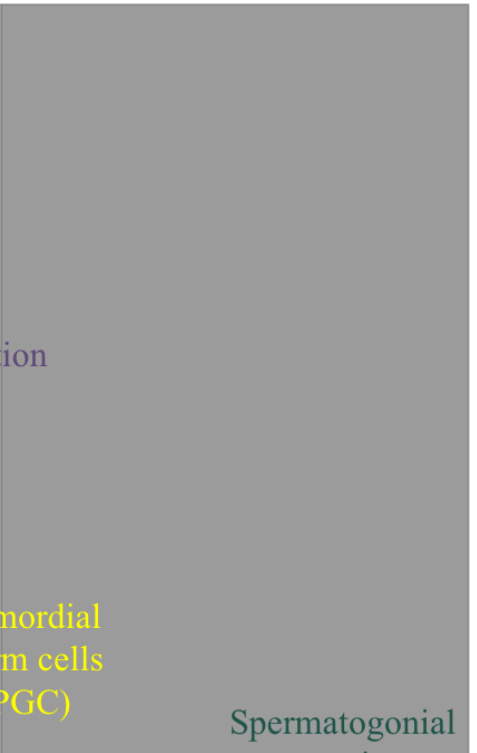

progenitors

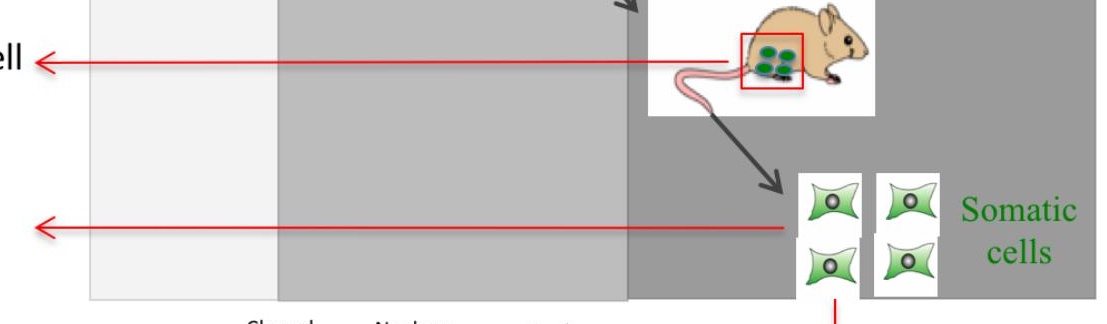

NT-ESC

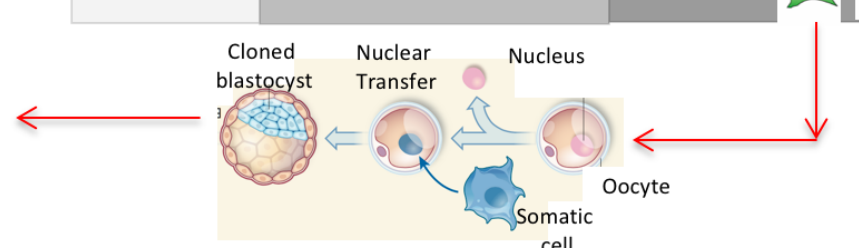

cell 
bioRxiv preprint doi: https://doi.org/10.1101/030676; this version posted December 12, 2015. The copyright holder for this preprint (which was not certified by peer review) is the author/funder, who has granted bioRxiv a license to display the preprint in perpetuity. It is made available under aCC-BY-NC-ND 4.0 International license.

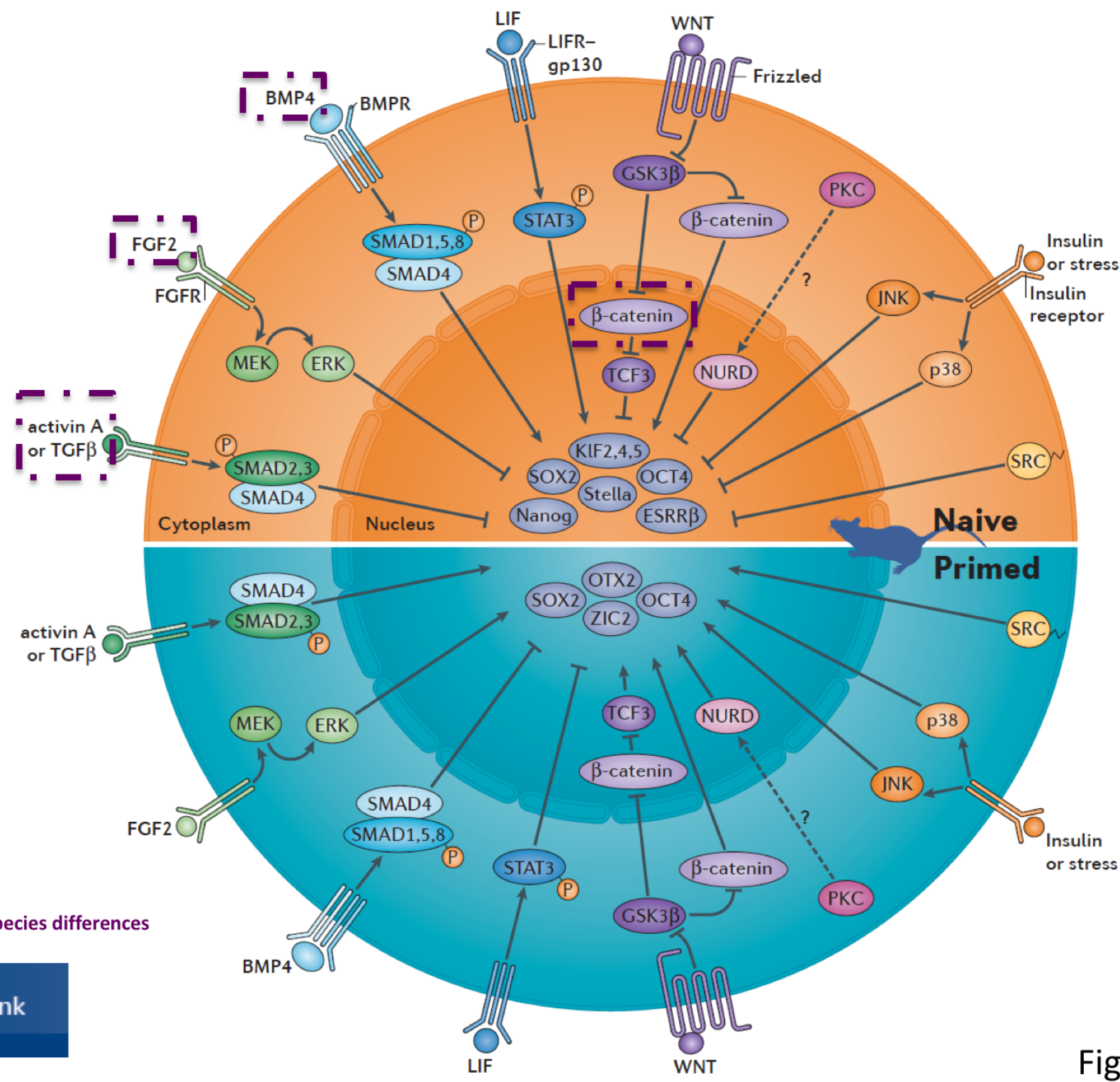

Figure 2 
bioRxiv preprint doi: https://doi.org/10.1101/030676; this version posted December 12, 2015. The copyright holder for this preprint (which was not certified by peer review) is the author/funder, who has granted bioRxiv a license to display the preprint in perpetuity. It is made available under aCC-BY-NC-ND 4.0 International license.

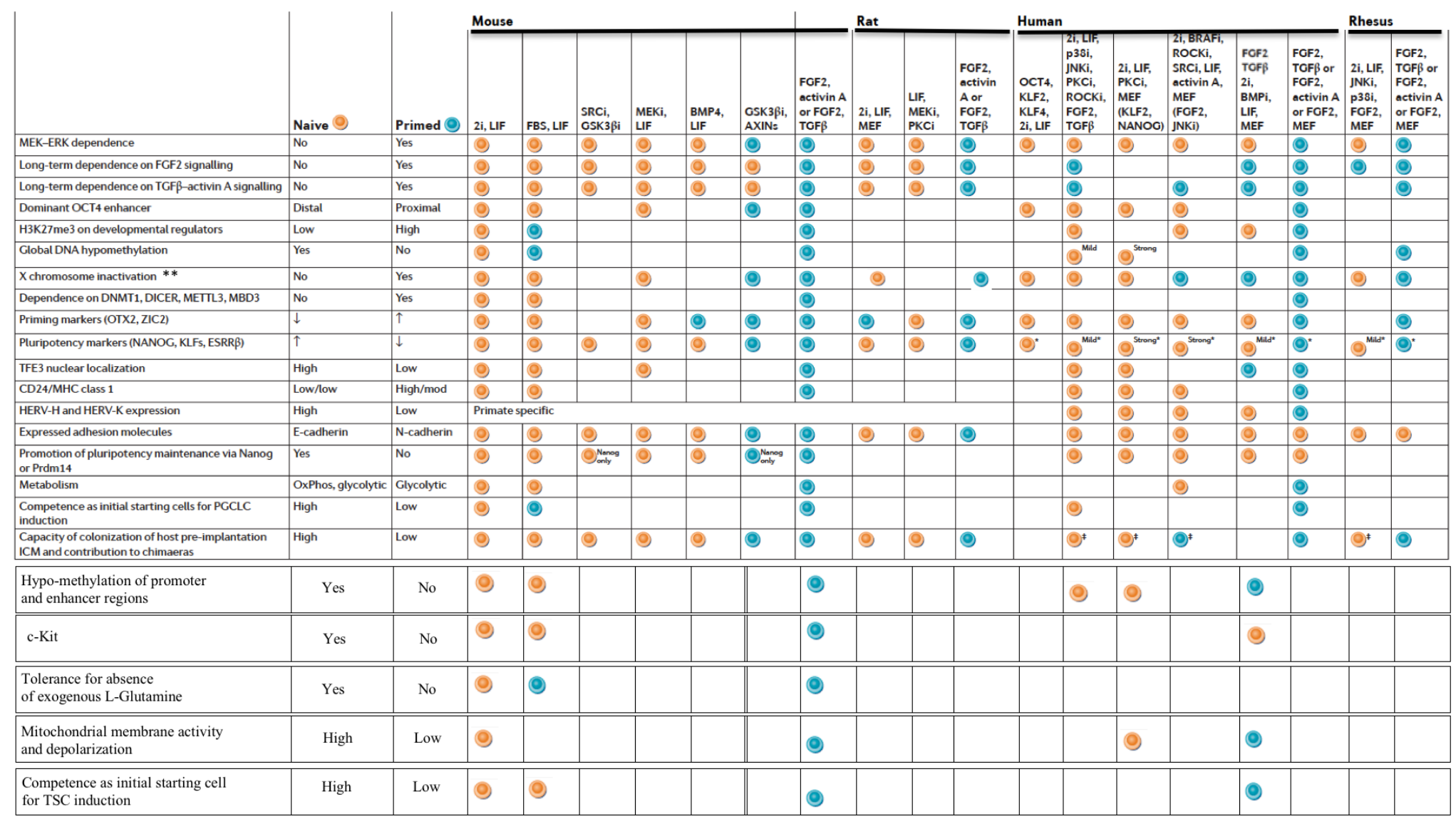

Comments: Empty boxes - results have not been reported thus far

** Note this classification presents over-simplification of $\mathrm{X}$ chromosome active and inactive conformations that can be assumed, particularly in non-rodent cell lines. 
bioRxiv preprint doi: https://doi.org/10.1101/030676; this version posted December 12, 2015. The copyright holder for this preprint (which was not certified by peer review) is the author/funder, who has granted bioRxiv a license to display the preprint in perpetuity. It is made available under aCC-BY-NC-ND 4.0 International license.

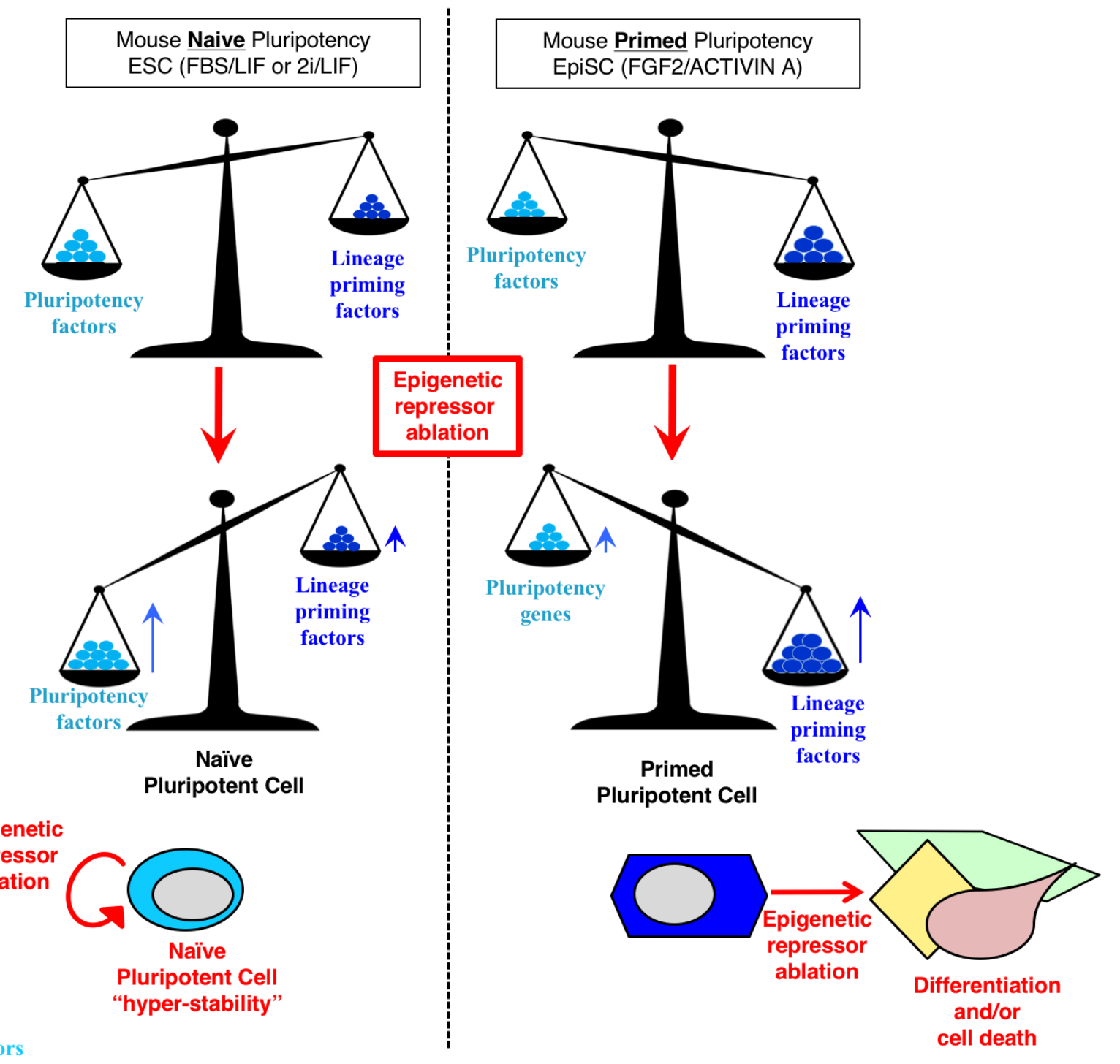

Pluripotency factors

Lineage priming \& anti-apoptotic factors 
bioRxiv preprint doi: https://doi.org/10.1101/030676; this version posted December 12,2015 . The copyright holder for this preprint (which was not certified by peer review) is the author/funder, who has granted bioRxiv a license to display the preprint in perpetuity. It is made available under aCC-BY-NC-ND 4.0 International license.

a

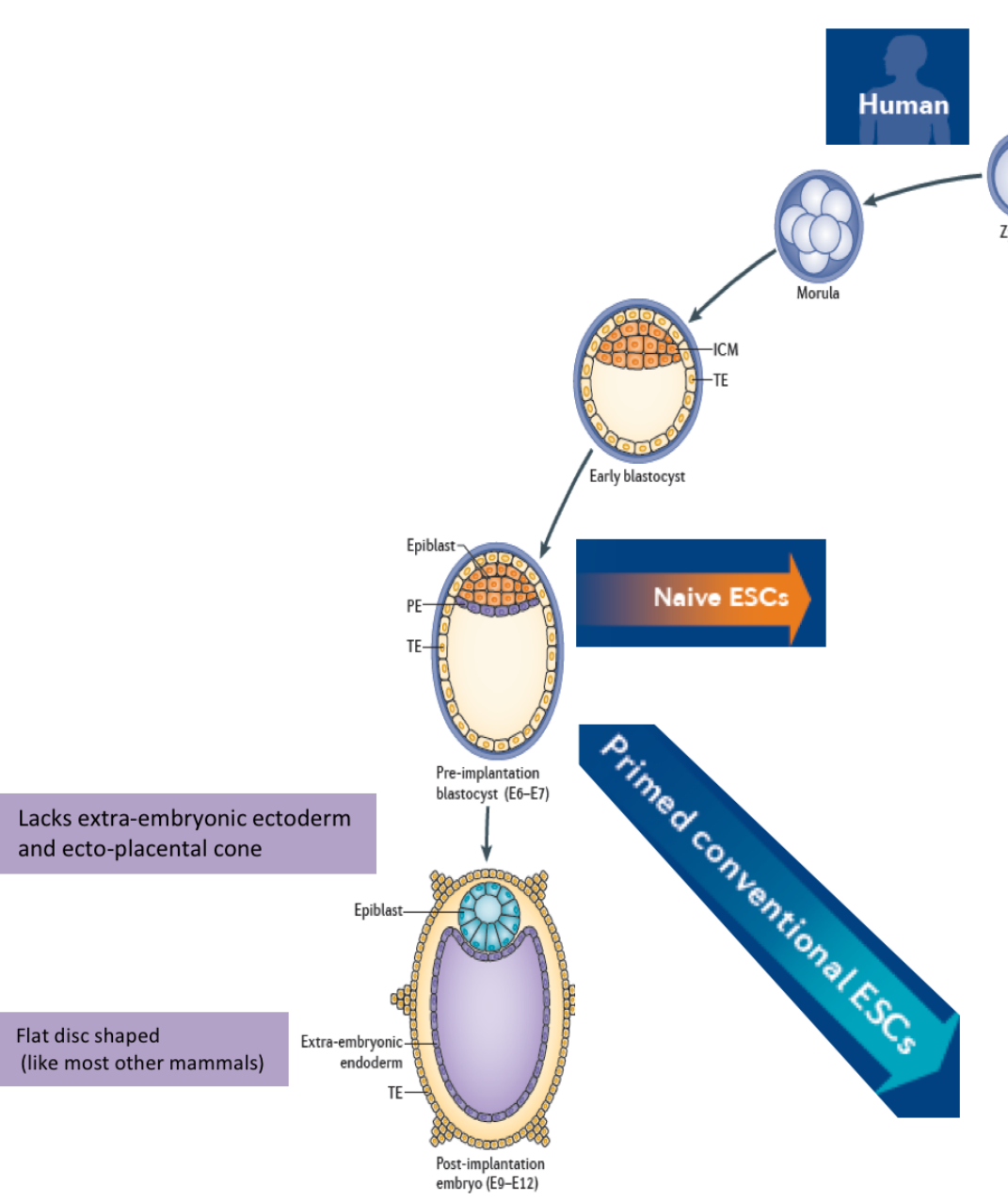

b

Gene expression in pre-implantation epiblasts

Gene Human Mouse

KLF2 No Yes

KLF17 Yes No

ERAS No Yes

XIST Low No

DNMT3L High Low

ESRRB No Yes

BMP4 No Yes

NODAL Yes No 
bioRxiv preprint doi: https://doi.org/10.1101/030676; this version posted December 12,2015 . The copyright holder for this preprint (which was not certified by peer review) is the author/funder, who has granted bioRxiv a license to display the preprint in perpetuity. It is made available under aCC-BY-NC-ND 4.0 International license.

C

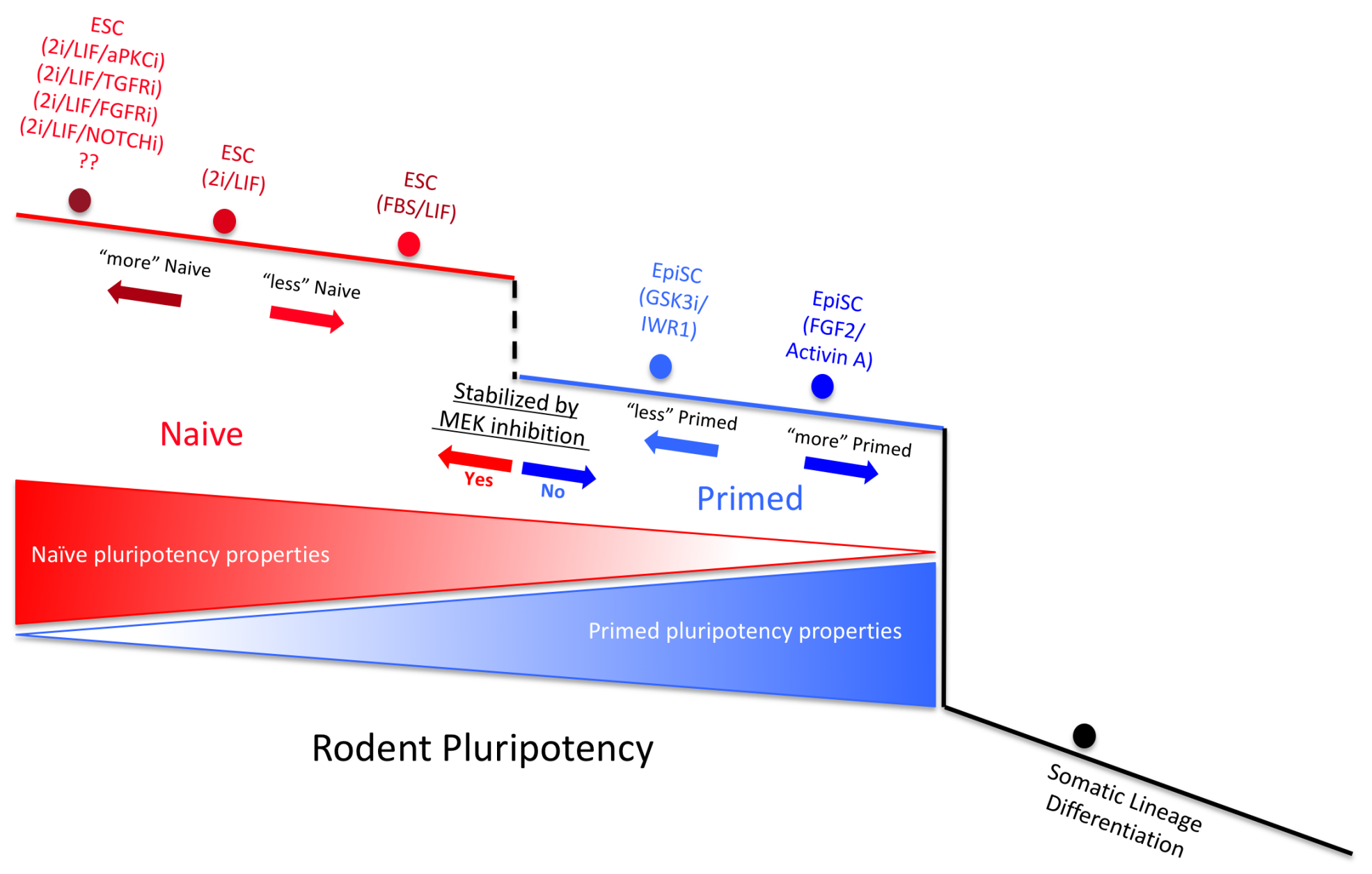

Figure 5 